\title{
Investigation on the Drag Coefficient of the Steady and Unsteady Flow Conditions in Coarse Porous Media
}

Hadi Norouzi ( $\square$ hadinorouzi72@gmail.com )

University of Zanjan https://orcid.org/0000-0001-6082-3736

Jalal Bazargan

University of Zanjan

Faezah Azhang

University of Zanjan

Rana Nasiri

University of Zanjan

\section{Research Article}

Keywords: Drag Coefficient, Friction Coefficient, Hydraulic Gradient, Porous Media, Steady and Unsteady Flow

Posted Date: March 24th, 2021

DOI: https://doi.org/10.21203/rs.3.rs-332975/v1

License: (c) (i) This work is licensed under a Creative Commons Attribution 4.0 International License. Read Full License 
1 Investigation on the Drag Coefficient of the Steady and Unsteady Flow Conditions in

Coarse Porous Media

Hadi Norouzi ${ }^{\text {a* }}$, Jalal Bazargan ${ }^{\text {b }}$, Faezeh Azhang ${ }^{\text {c, Rana Nasiri }}{ }^{d}$

a. PhD Candidate of Hydraulic Structures, Department of Civil Engineering, University of Zanjan, Zanjan, Iran (hadinorouzi72@gmail.com)

b. Associate Professor, Department of Civil Engineering, University of Zanjan, Zanjan, Iran

c. Masters of Hydraulic Structures, Department of Civil Engineering, University of Zanjan, Zanjan, Iran (faezehazhang73@yahoo.com)

d. Masters of Hydraulic Structures, Department of Civil Engineering, University of Zanjan, Zanjan, Iran (․ㅗsiri.rana@znu.ac.ir)

\section{Abstract}

The study of the steady and unsteady flow through porous media and the interactions between fluids and particles is of utmost importance. In the present study, binomial and trinomial equations to calculate the changes in hydraulic gradient (i) in terms of flow velocity (V) were studied in the steady and unsteady flow conditions, respectively. According to previous studies, the calculation of drag coefficient $\left(\mathrm{C}_{d}\right)$ and consequently, drag force $\left(\mathrm{F}_{d}\right)$ is a function of coefficient of friction (f). Using Darcy-Weisbach equations in pipes, the hydraulic gradient equations in terms of flow velocity in the steady and unsteady flow conditions, and the analytical equations proposed by Ahmed and Sunada in calculation of the coefficients a and $b$ of the binomial equation and the friction coefficient (f) equation in terms of the Reynolds number $(\mathrm{Re})$ in the porous media, equations were presented for calculation of the friction coefficient in terms of the Reynolds number in the steady and unsteady flow conditions in 1D 
24 (one-dimensional) confined porous media. Comparison of experimental results with the

46 results of the proposed equation in estimation of the drag coefficient in the present study confirmed the high accuracy and efficiency of the equations. The mean relative error (MRE) between the computational (using the proposed equations in the present study) and observational (direct use of experimental data) friction coefficient for small, medium and large grading in the steady flow conditions was equal to $1.913,3.614$ and $3.322 \%$, respectively. In the unsteady flow condition, the corresponding values of 7.806, 14.106 and $10.506 \%$ were obtained, respectively.

Keywords: Drag Coefficient, Friction Coefficient, Hydraulic Gradient, Porous Media, Steady and Unsteady Flow.

\section{Introduction}

Coarse-grained gravels (rockfill materials) have numerous applications in engineering including filtration, construction of gabions, lining of channels, stilling basins, ponds, and cobble stone dams as well as flood control.

In the fine-grained media, there is laminar flow with a linear relation between the hydraulic gradient and the flow velocity and flow follows Darcy's law (Eq. 1) (McWhorter et al. 1977). However, in the coarse-grained media, due to the presence of voids, the velocity of the flow is high with the flow tendency to turbulence (Hansen et al. 1995), with a nonlinear relation between the hydraulic gradient and flow velocity and low follows non-Darcy law. The equations for the calculation of hydraulic gradient in the non-Darcy media in the steady flow condition are classified into two groups of power and binomial equations, according to Eqs. 2 and 3 (Forchheimer, 1901; Leps, 1973; Stephenson, 1979).

$$
\mathrm{i}=\left(\frac{1}{\mathrm{k}}\right) \mathrm{V}
$$


$48 \quad i=a V+b V^{2}$

49 The binomial equation was proved by dimensional analysis by Ward (1964) and by the

50 Navier-Stokes equations by Ahmed and Sunada (1969) and has a higher accuracy and

51 efficiency in comparison to the exponential equation (Stephenson 1979, Leps 1973).

52 Ergun (1952) studied the coefficients of the binomial equation of the hydraulic gradient by 53 passing nitrogen gas through a cylinder with an area of $7.24 \mathrm{~cm}^{2}$ that was filled with 54 aggregate and presented Eq. (4) to calculate the coefficients a, b.

$55 \quad a=150 \frac{v(1-n)^{2}}{d^{2} g n^{3}} \quad, b=1.75 \frac{(1-n)}{g d n^{3}}$

56 Ward (1964) presented Eq. (5), which can be proved using dimensional analysis, to calculate

57 the coefficients $a$ and $b$ in the free surface porous media.

$58 \quad a=\frac{v}{g k} \quad, b=\frac{C_{W}^{\prime}}{g \sqrt{k}}$

59 Kovacs (1980) studied a set of data with a Reynolds number range of 10 to 100 (according to

60 his definition of the Reynolds number) and presented an equation similar to that of Ergun 61 (Eq. (6)).

$62 \quad a=\frac{144 v(1-n)^{2}}{g n^{3} d^{2}} \quad, b=\frac{2.4(1-n)}{g n^{3} d}$

63 Ahmed and Sunada (1969) presented Eq. (7) to calculate the coefficients a and b using the 64 Navier-Stokes equations.

65

$$
a=\frac{\mu}{\rho g k} \quad, b=\frac{1}{g \sqrt{c k}}
$$

$$
K=C d^{2}
$$


66 Ergun-Reichelt presented an equation for calculation of the coefficients a, b (Eq. (8)) (Fand

67 and Thinakaran, 1990).

$68 \quad a=214 \frac{M^{2}(1-n)^{2} v}{g n^{3} d^{2}} \quad, b=1.57 \frac{M(1-n)}{g n^{3} d} \quad, M=1+\frac{2}{3} \frac{d}{D(1-n)}$

69 Equations (4) to (8) and several equations including those proposed by (Muskrat 1937;

70 Engelund 1953; Irmay 1958; Stephenson 1979; Jent 1991; Kadlec and Knight 1996;

71 Sidiropoulou et al. 2007; Sedghi and Rahimi 2011) were presented to calculate the 72 coefficients of the binomial equation $(a, b)$ in steady flow conditions. A semi-analytical 73 solution of the nonlinear differential equations constructing a fully saturated porous medium 74 is presented by (Abbas et al. 2021).

75 A comprehensive equation with respect to the effects of unsteady flow conditions was 76 proposed by Polubarinova-Kochino (1952) (Eq. (9)) (Hannoura and McCorcoudale, 1985).

$77 \quad i=a V+b V^{2}+c\left(\frac{d V}{d t}\right)$

78 Where the coefficient of the third term (c) is obtained using Eq. (10).

$79 \quad c=\frac{n+C_{m}(1-n)}{n g}$

80 Where $\mathrm{C}_{\mathrm{m}}$ represents the proportion of fluid that vibrates with the vibration of the particle. In

81 other words, $\mathrm{C}_{\mathrm{m}}$ is the added mass coefficient.

82 Hannoura and McCorquodale (1985) performed an experimental study and indicated that $\mathrm{C}_{\mathrm{m}}$

83 was insignificant and negligible. In other words, by removing $C_{m}$ from Eq. (10), the third

84 term of Eq. (9) can be expressed as Eq. (11).

$85 \quad c\left(\frac{d V}{d t}\right)=\frac{1}{g}\left(\frac{d V}{d t}\right)$ 
where $\mathrm{V}$ is flow velocity $(\mathrm{m} / \mathrm{s}), \mathrm{k}$ is hydraulic conductivity $(\mathrm{s} / \mathrm{m}), \mathrm{i}$ is hydraulic gradient, $\mathrm{m}$ and $\mathrm{n}$ are values dependent on the properties of the porous media, fluid and flow, while a and $\mathrm{b}$ are coefficients that are dependent on the properties of the porous media as well as the fluid.

Shokri et al. (2011) experimentally studied unsteady flow in a free surface coarse-grained porous media and concluded that the third term $\left(c\left(\frac{d V}{d t}\right)\right)$ has insignificant effect on the accuracy of calculations.

In the present study, the binomial (Eq. 3) and trinomial (Eq. 8) equations were used to calculate the changes in hydraulic gradient in terms of velocity in steady and unsteady flow conditions, respectively.

The simulated annealing algorithm is often used for water resources management, model calibration, decision making, etc. (Bechler et al. 2013; Cao and Ye 2013; Jiang et al. 2018). (Hu et al. 2019) Kriging-approximation simulated annealing (KASA) optimization algorithm has been used to optimize the flow parameters in the porous media.

Particle swarm optimization (PSO) algorithm is a population-based evolutionary algorithm and is used in civil engineering and water resources optimization problems such as reservoir performance (Nagesh Kumar and Janga Reddy, 2007), water quality management (Afshar et al. 2011, Lu et al. 2002, Chau, 2005) and optimization of the Muskingum method coefficients (Chu and Chang 2009, Moghaddam et al. 2016, Bazargan and Norouzi 2018, Norouzi and Bazargan 2020, Norouzi and Bazargan 2021). Therefore, in the present study, the particle swarm optimization (PSO) algorithm was used to optimize the coefficients of the Forchheimer binomial equation (Eq. 3) as well as the Polubarinova-Kochino trinomial equation (Eq. 8). 
109 In addition to the above classification, flow in the coarse-grained media can be classified into

110 the following two general groups.

111 A: Free surface flow through and on coarse-grained layers such as gabions and gravel dams

112 in which the flow is in contact with the free environment on one side.

113 B: Confined flow through coarse-grained layers such as coarse-grained filters of rockfill

114 dams and coarse-grained layers confined between concrete elements and fine materials of

115 hydraulic structures. In such layers, flow from all sides is in contact with an impermeable

116 layer or a layer with low permeability in comparison to gravel materials.

117 The study of flow through confined porous media is of great importance in geology (Lei et al.

118 2017), petroleum (Song et al. 2014) and industry (Rahimi et al. 2017). Flow through

119 pressurized porous media has been studied experimentally and numerically by (Ingham and

120 Pop 2005; Sheikh and Pak 2015; and Zhu et al. 2016). The study of fluid flow in porous

121 media is performed by a network of capillary pipes with a microscopic approach by (Hoang

122 et al. 2013). (Hsu and Chen 2010) proposes a multiscale flow and transport model in three-

123 dimensional fractal random fields for use in porous media.

124 Estimation of drag force due to flow-aggregate interactions is very important in modeling of 125 confined porous media (Sheikh and Qiu, 2018). Pore-scale drag and relative motion between

126 fluid and aggregates are important due to the formation of non-uniform velocity and

127 consequently, the formation of non-uniform force (Sheikh and Qiu, 2018). (Ergun 1952; Wen

128 and Yu 1966; Di Felice 1994; Schlichting and Gersten 2000; Hill et al. 2001a; Hill et al.

129 2001b; Van der Hoef et al. 2005; Bird et al. 2007; Yin and Sundaresan 2009; Zhang et al.

130 2011; Rong et al. 2013) studied the drag force in the confined porous media considering a

131 certain number of particles. The flow regime and drag force of a single particle are different

132 from those of interactive particles (Zhu et al. 1994; Liang et al. 1996; Chen and Wu 2000). 
133 The accuracy and efficiency of the presented empirical equations for calculation of the drag

134 force have not been evaluated and compared for a wide range of porosity and Reynolds

135 numbers. In other words, the relation between drag force and Reynolds number has been

136 studied experimentally or numerically in previous studies. Accurate calculation of drag

137 coefficient and drag force and its application in one-dimensional analysis of steady flow in

138 porous media (gradually varied flows) has increased the accuracy of calculations, especially

139 in sections with high curvature of the longitudinal profile of water surface (Gudarzi et al.

140 2020). To predict the flow characteristics in porous media, it is very important to acquire the

141 structures of the porous media. However, it is difficult to measure the three-dimensional

142 microstructures of high-resolution porous media due to the expensive cost of the equipment

143 (Zhang et al. 2016).

144 Since the drag force $\left(\mathrm{F}_{d}\right)$ is a function of the drag coefficient $\left(\mathrm{C}_{d}\right)$ and $\mathrm{C}_{\mathrm{d}}$ is a function of the

145 friction coefficient (f), using binomial and trinomial equations, Darcy-Wiesbach equation,

146 Ahmed and Sunada analytical equation to calculate coefficients $a, b$ and changes of 147 coefficient $\mathrm{f}$ in terms of the Reynolds number $(\mathrm{Re})$ in porous media, equations were presented 148 in the present study to calculate $\mathrm{f}$ in terms of Re in steady and unsteady flow conditions in 1D 149 confined porous media. Comparison of the results of the proposed equations with 150 experimental data indicated the high accuracy and efficiency of the proposed equations. In 151 other words, in the present study, equations were presented for steady and unsteady flow 152 conditions in porous media and applicable for any number of particles as well as all Reynolds 153 numbers.

\section{Materials and methods}

\section{$155 \quad 2.1$ Experimental data}


156 The experiments were performed in the Laboratory of the Faculty of Civil Engineering of

157 Zanjan University, Zanjan, Iran on a steel cylinder with a diameter of $16 \mathrm{~cm}$ and a length of

$15870 \mathrm{~cm}$, of which $40 \mathrm{~cm}$ was filled with aggregates considering steady and unsteady flow

159 conditions. In order to develop unsteady flow condition using the gravity method, a tank was

160 installed at a height of 13 meters above the cylinder inlet on the roof of the faculty. To

161 discharge the cylinder outflow, a tank was installed in the laboratory. Using a camcorder,

162 water height in the discharge tank was recorded at different times and then, using the

163 volumetric method, discharge of the unsteady flow was recorded at different times. To

164 measure water depth, piezometers were installed at the beginning and end of the cylinder.

165 The tanks used, aggregates in three gradations (small, medium and large) and the steel

166 cylinder are shown in Figure 1. The characteristics of the aggregates and gradation curves of

167 the three gradations (small, medium and large) are shown in Table 1 and Fig. 2, respectively.

168 Figure 3 and Figure 4 show changes in hydraulic gradient (i) versus flow velocity (V) for all

169 three types of small, medium and large grained materials in the steady and unsteady flow

170 conditions, respectively.

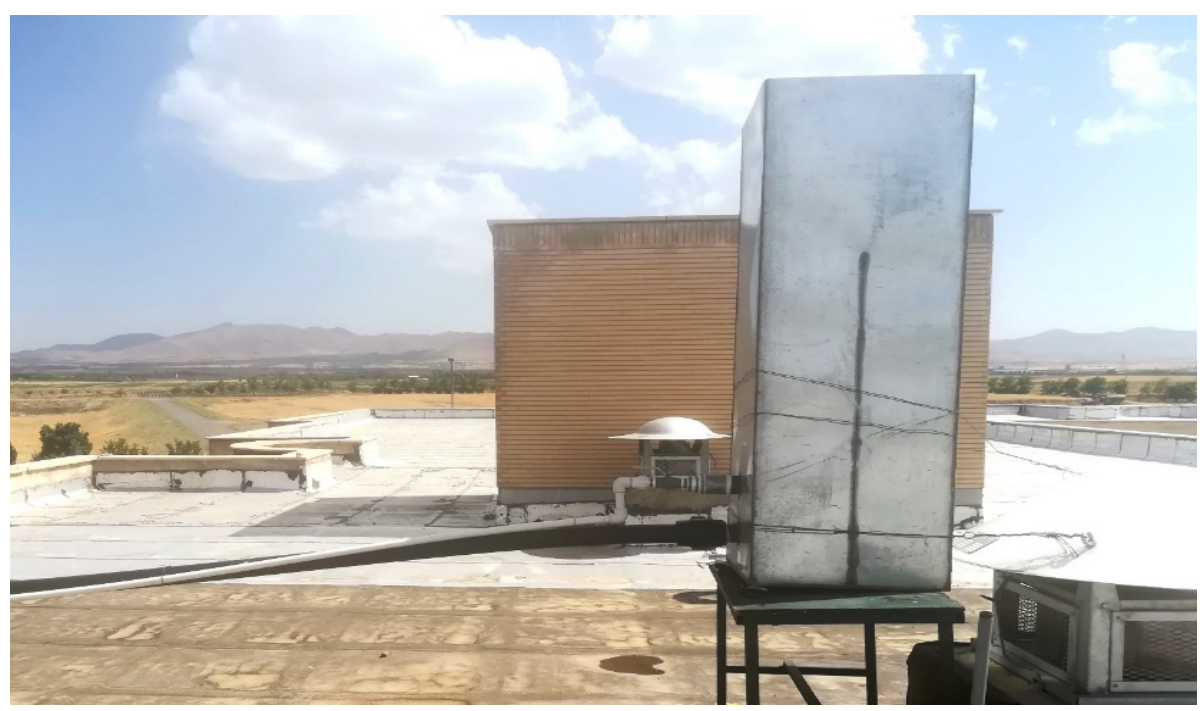



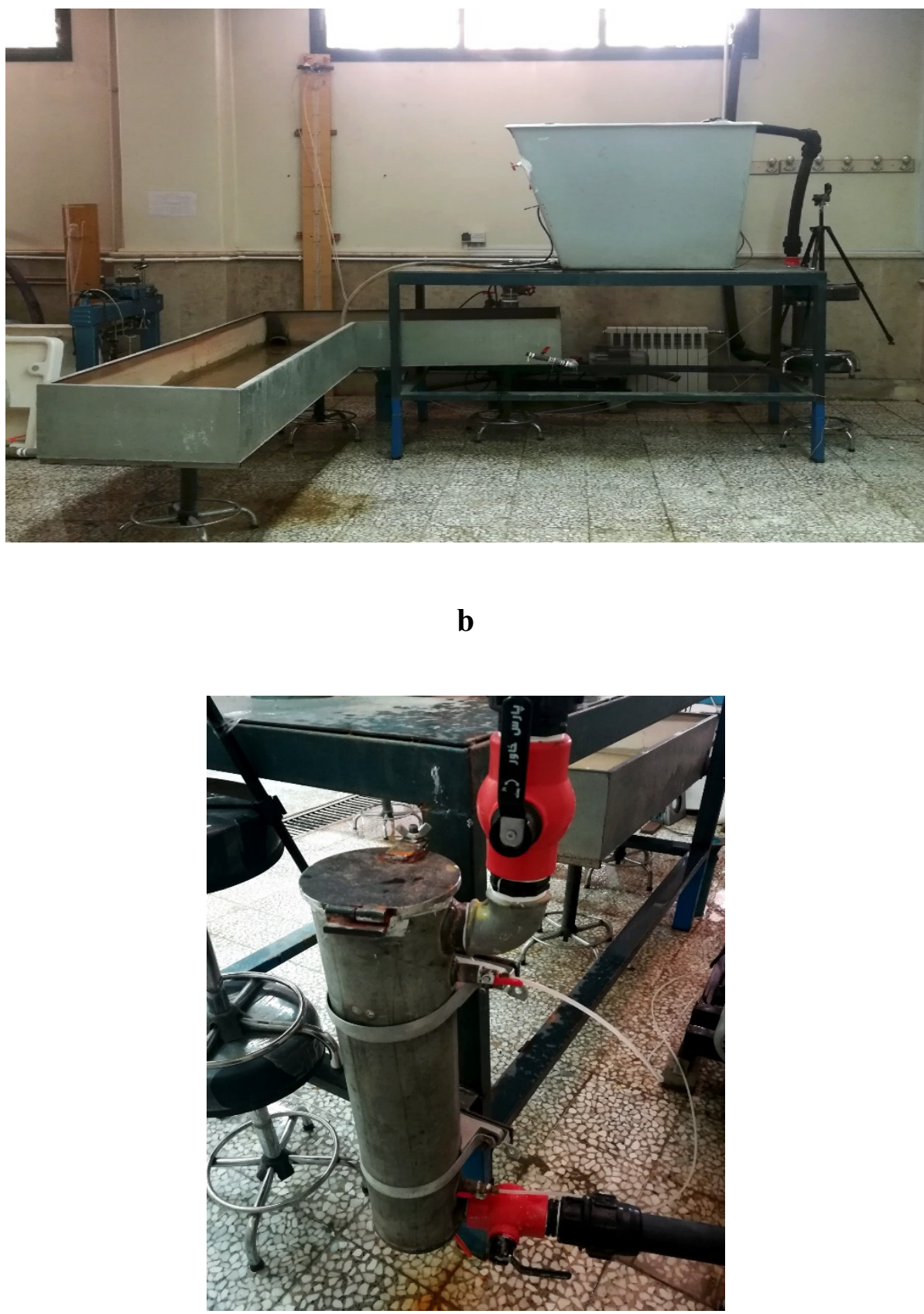

177 Figure 1. Schematic view of the experimental setup, a) the installed tank on the roof, b) the 
Table 1. Characteristics of the experimental materials

\begin{tabular}{cccccccccc}
\hline Materials & $\begin{array}{c}\mathbf{d}_{\mathbf{0}} \\
(\mathbf{m m})\end{array}$ & $\begin{array}{c}\mathbf{d}_{\mathbf{1 0}} \\
(\mathbf{m m})\end{array}$ & $\begin{array}{c}\mathbf{d}_{\mathbf{3 0}} \\
(\mathbf{m m})\end{array}$ & $\begin{array}{c}\mathbf{d}_{\mathbf{5} 0} \\
(\mathbf{m m})\end{array}$ & $\begin{array}{c}\mathbf{d}_{\mathbf{6} 0} \\
(\mathbf{m m})\end{array}$ & $\begin{array}{c}\mathbf{d}_{\mathbf{1 0 0}} \\
(\mathbf{m m})\end{array}$ & $\mathbf{C}_{\mathbf{u}}$ & $\mathbf{C}_{\mathbf{c}}$ & porosity \\
\hline Small & 4 & 7 & 10 & 12.5 & 14.5 & 22 & 1.69 & 0.69 & 0358 \\
Medium & 4.75 & 14.5 & 18.5 & 19 & 21.3 & 32 & 1.16 & 0.95 & 0.410 \\
Large & 12.7 & 22.7 & 23.9 & 22 & 25.05 & 32 & 1.11 & 0.99 & 0.448 \\
\hline
\end{tabular}

183

184 In Table 1 , the coefficient of uniformity $\left(\mathrm{C}_{\mathrm{u}}\right)$ and the coefficient of curvature $\left(\mathrm{C}_{\mathrm{c}}\right)$ were equal 185 to $\frac{D_{60}}{D_{10}}$ and $\frac{\left(D_{30}\right)^{2}}{D_{10} * D_{60}}$, respectively.

186

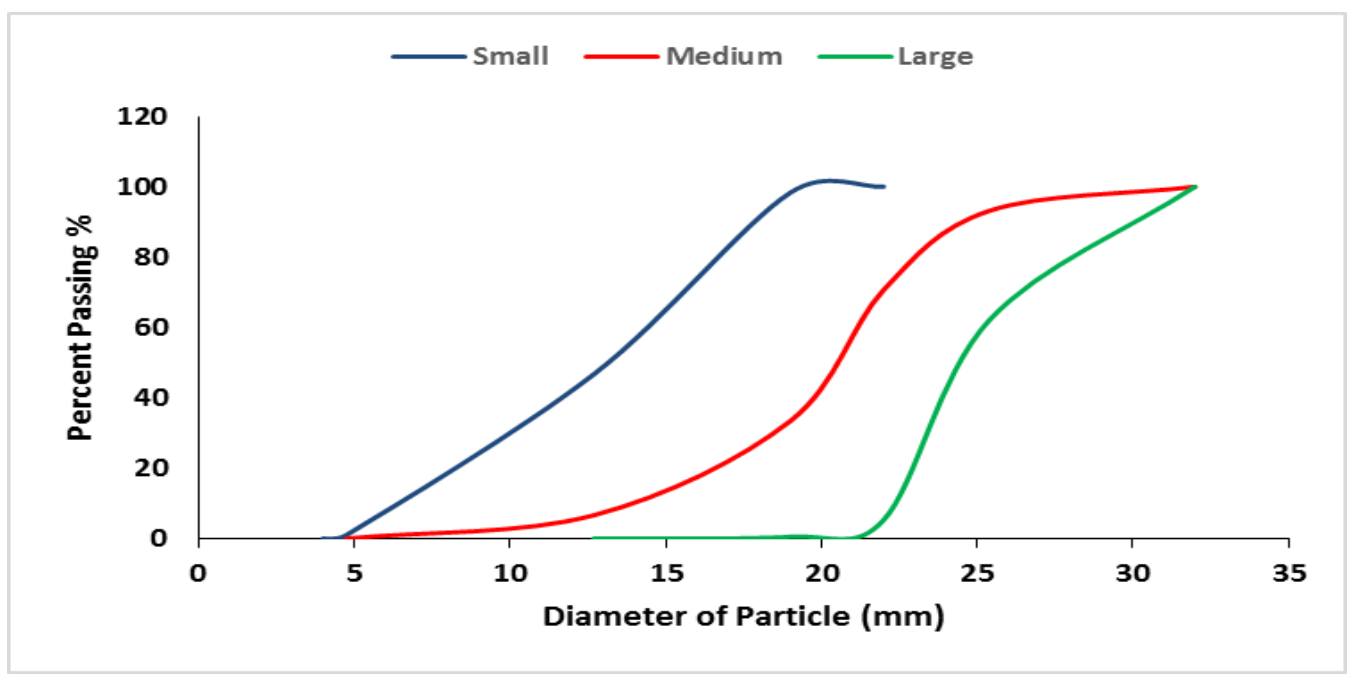

187

Figure 2. Gradation curve of different materials

188

189

190

191

192 


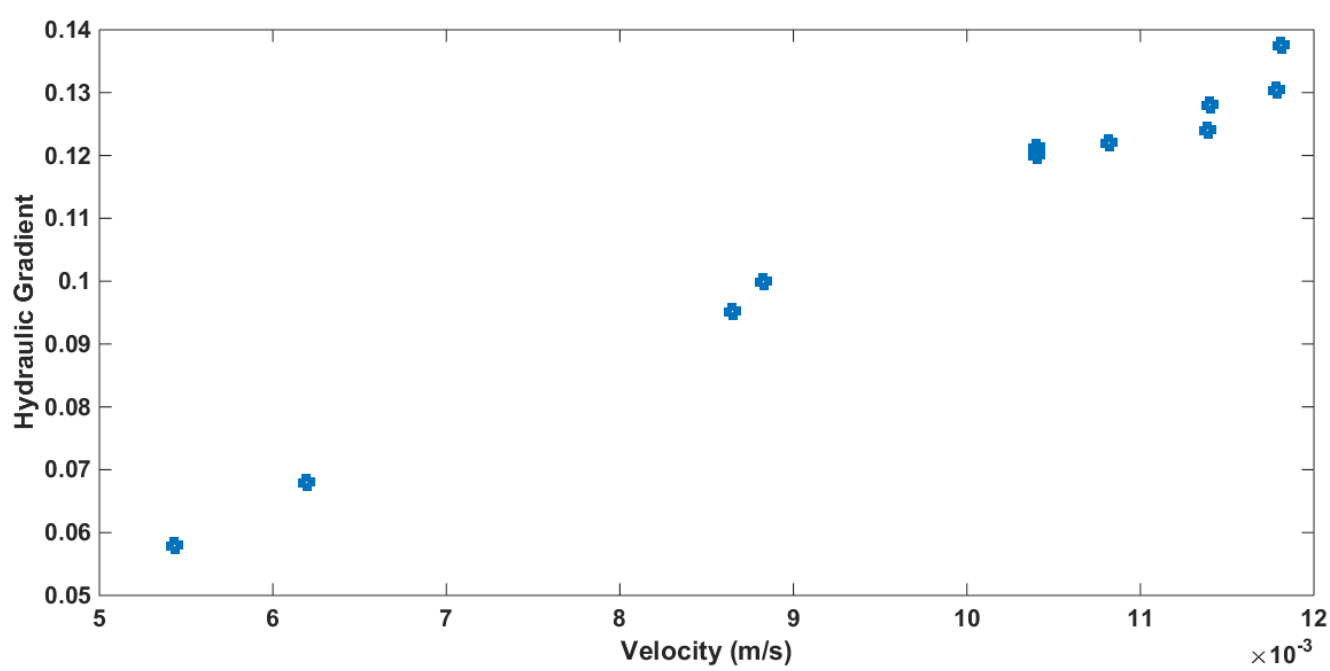

194

a. Small

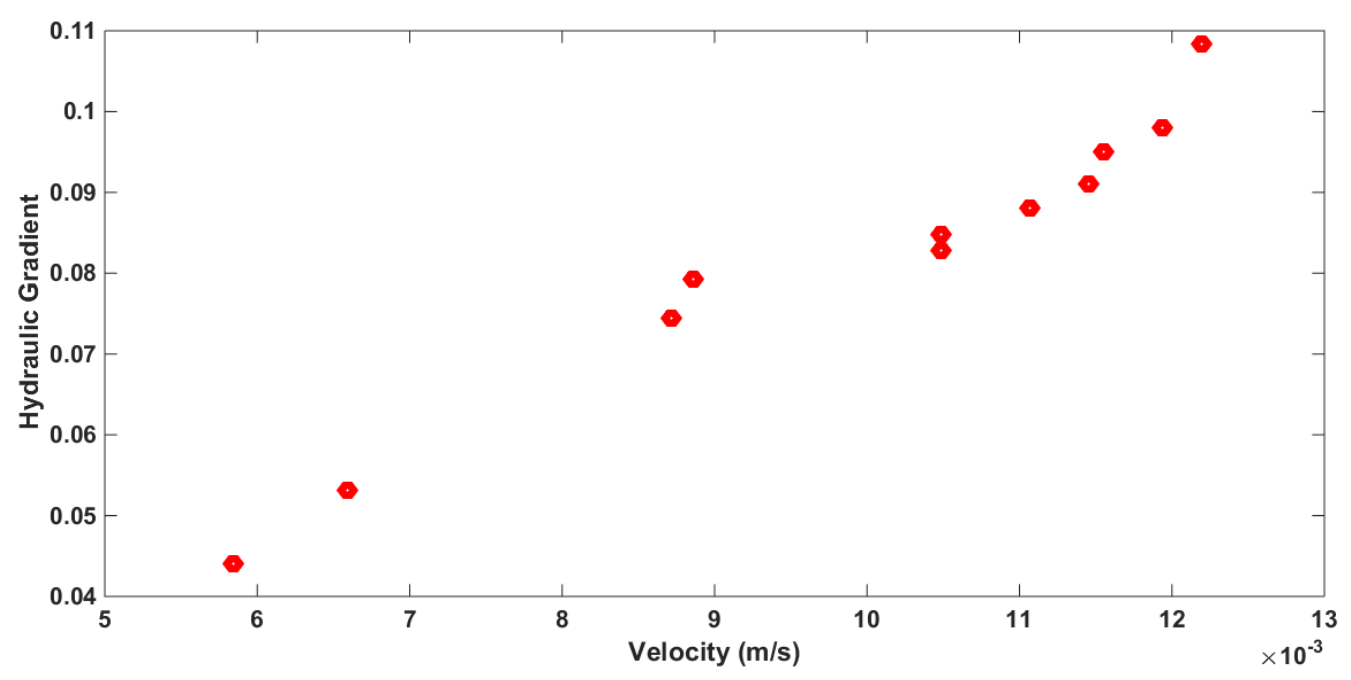

b. Medium

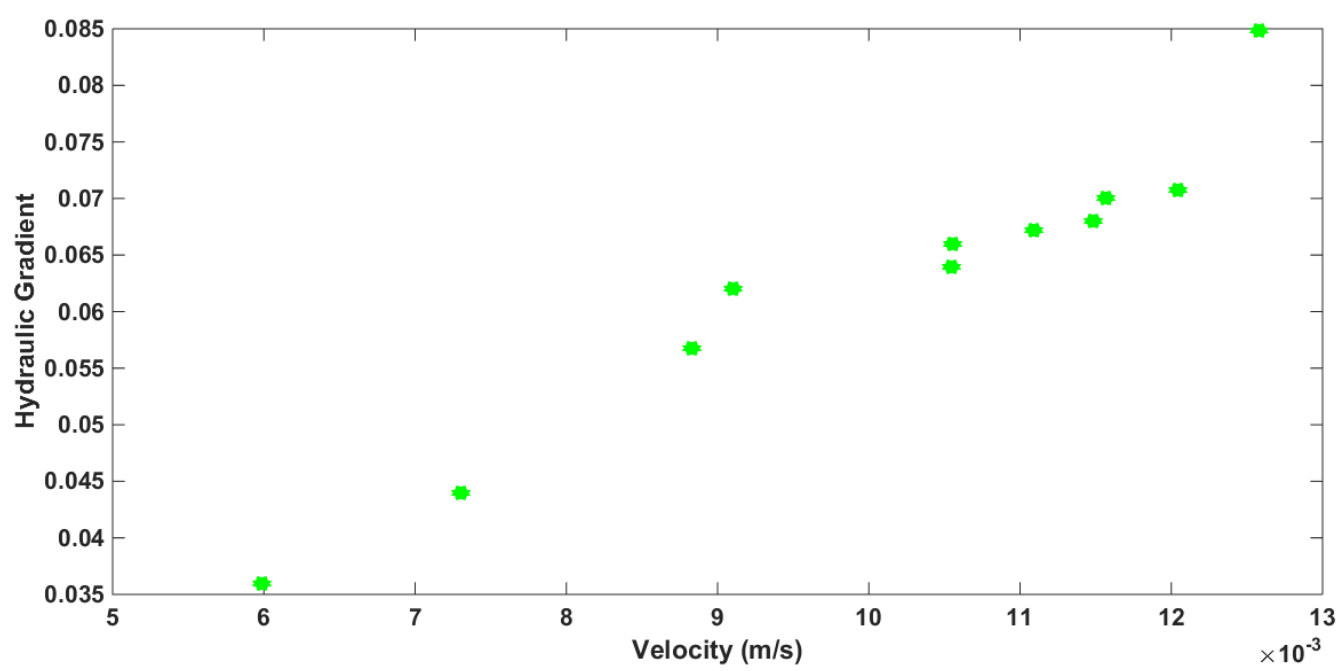

c. Large

Figure 3. Changes in hydraulic gradient versus steady flow velocity recorded in the laboratory 


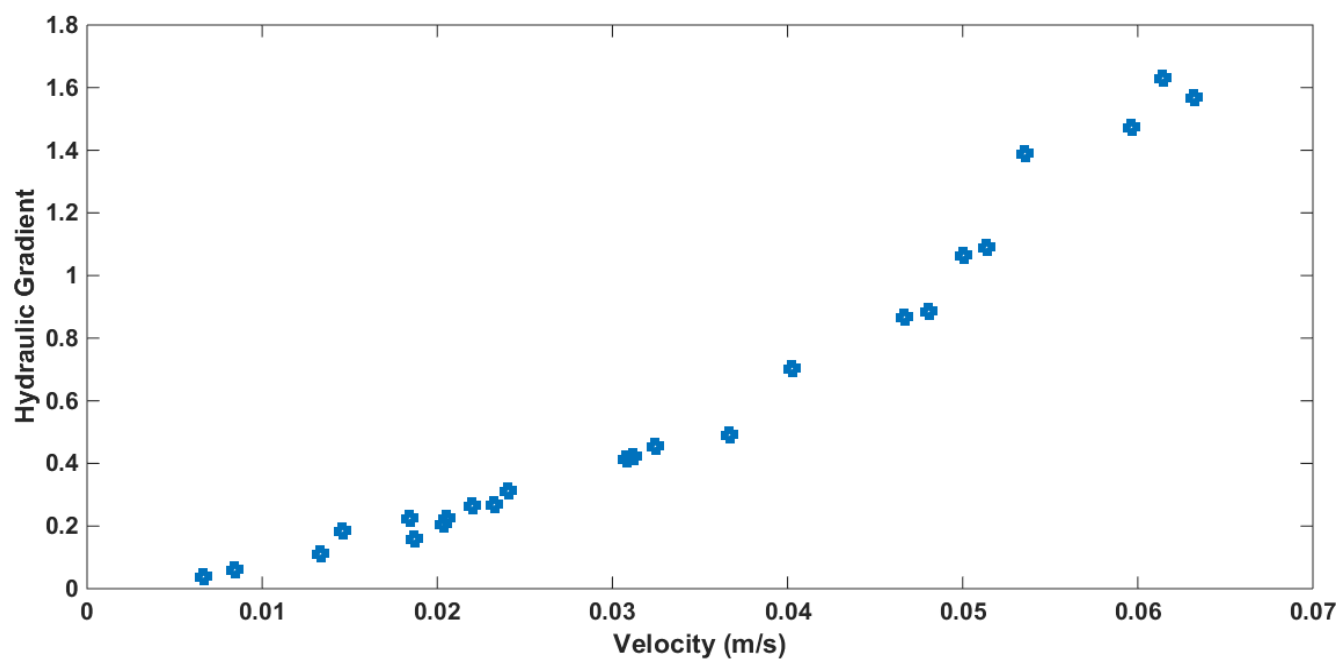

a. Small

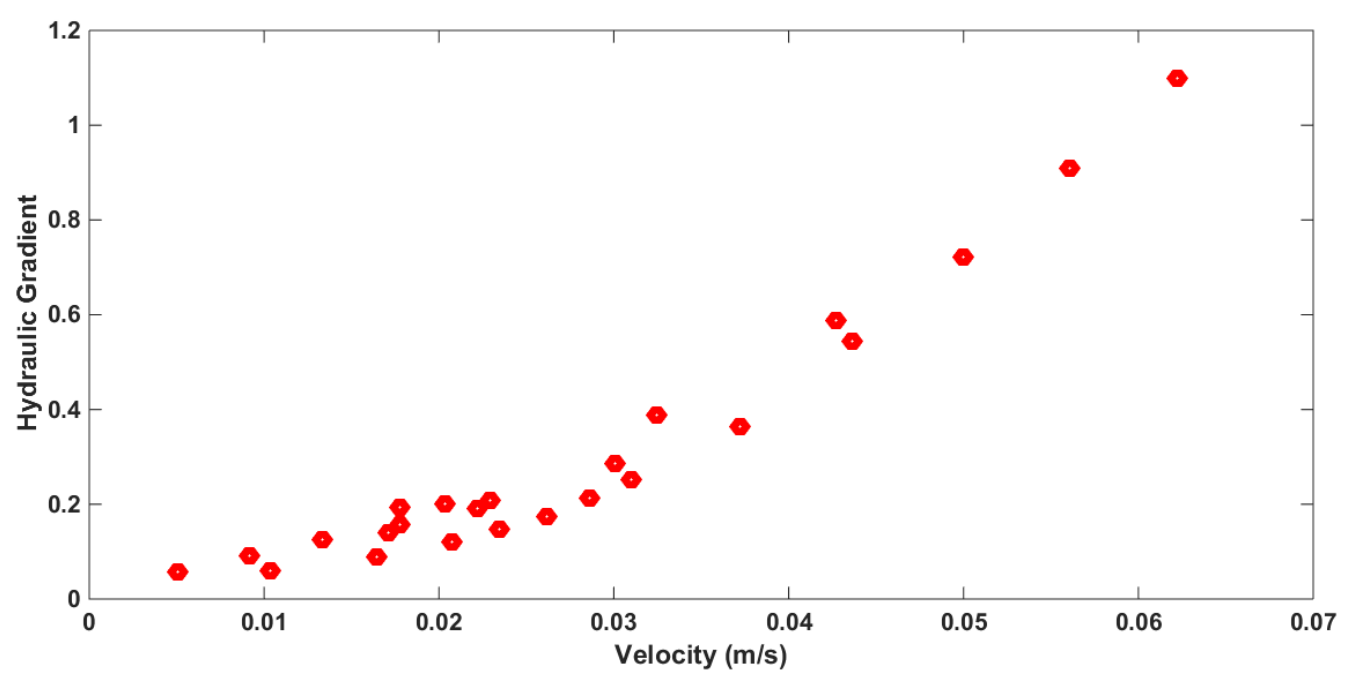

b. Medium

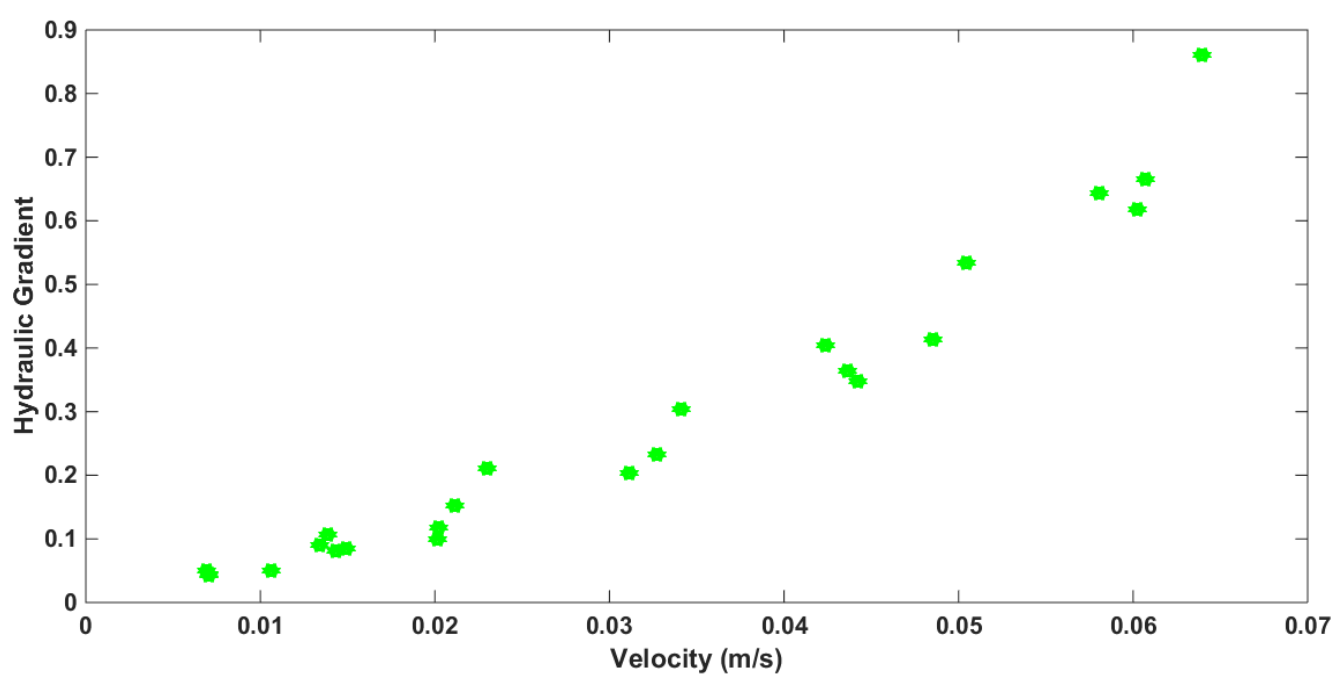

c. Large

Figure 4. Changes in hydraulic gradient versus unsteady flow velocity recorded in the laboratory 


\subsection{Particle Swarm Optimization (PSO) Algorithm}

210 PSO method is also a population based evolutionary search optimization method inspired

211 from the movement of bird flock (swarm) (Eberhart and Kennedy 1995; Chau 2007; Clerc

212 and Kennedy 2002). The basic idea in PSO is based on the assumption that potential solutions

213 are flown through hyperspace with acceleration towards more optimum solutions. Each

214 particle adjusts its flying according to the experiences of both itself and its companions.

215 During the process; the overall best value attained by all the particles within the group and

216 the coordinates of each element in hyperspace associated with its previous best fitness

217 solution are recorded in the memory (Chau 2007; Kumar and Reddy 2007). The details of 218 PSO can be obtained elsewhere (Shi and Eberhart 1998; Clerc and Kennedy 2002; Chau 219 2007; Gurarslan and Karahan 2011; Karahan 2012; Di Cesare et al. 2015). Various 220 algorithms have been used to optimize the parameters of the Forchheimer equation and other 221 methods and other issues that need to be optimized, one of the fastest, most acceptable and 222 most widely used is the PSO algorithm, which has been confirmed by previous researchers. since in previous studies, efficiency, high convergence speed and proper accuracy of the PSO algorithm have been examined and approved, therefore, among different algorithms, the PSO algorithm is selected to optimize the Forchheimer equation coefficients (a, b) and

226 Polubarinova-Kochino equation coefficient (a, b, c).

227 To evaluate the optimum values of the binomial equation coefficients $(a, b)$ and the trinomial 228 equation coefficients (a, b, c), the minimization of the Mean Relative Error (MRE), which is 229 defined using Eq. (12), was used as the objective function in the Particle Swarm Optimization 230 algorithm.

231 $\operatorname{MRE}=\frac{1}{n} \sum_{i=1}^{n}\left|\frac{i_{i}-I_{i}}{i_{i}}\right| * 100$ 
232 Where $i_{i}$ and $I_{i}$ are observed and calculated values of hydraulic gradient using binomial and

233 trinomial equations, respectively. The flowchart used in the present study is presented in

234 Figure 5.

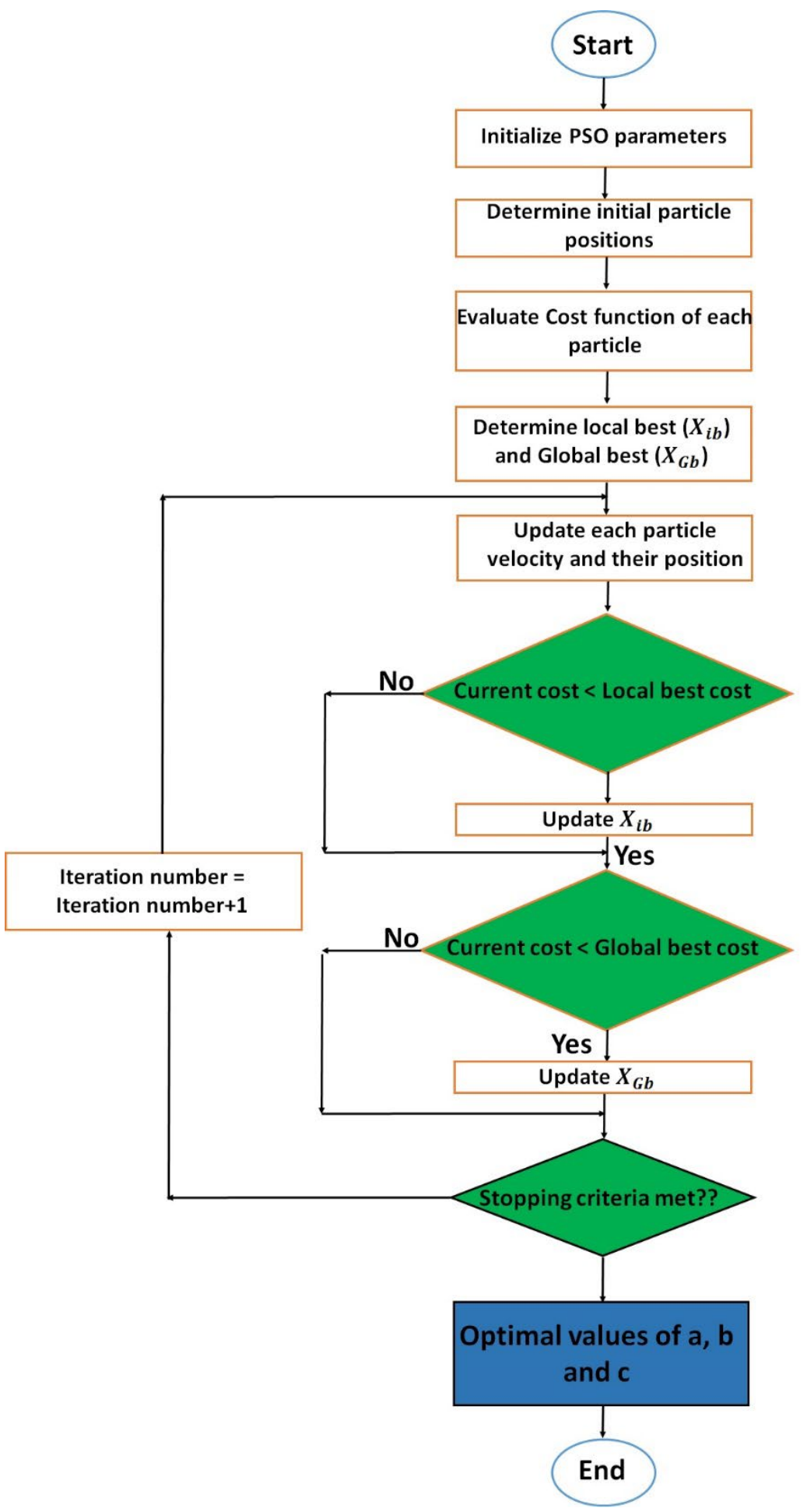

Figure 5. Particle Swarm Optimization (PSO) algorithm 


\subsection{Binomial (steady flow condition) and trinomial (unsteady flow condition) equations}

239 The hydraulic gradient in a coarse grained media is equal to the slope of the energy line $\left(\mathrm{S}_{\mathrm{f}}\right)$

240 (Stephenson 1969; Bari and Hansen 2002; Bazargan and Shoaei 2006). The calculation of

241 hydraulic gradient is of great importance in the steady flow (using the gradual variable flow

242 theory that analyzes flow one-dimensionally as well as the solution of the Parkin equation

243 that analyzes flow two-dimensionally) and unsteady flow (solving Saint-Venant equations)

244 analysis.

245 The binomial equation for the steady flow through porous media has been presented by

246 Forchheimer and equations for calculation of coefficients a and b were also presented using

247 dimensional analysis (Ward 1964) and Navier-Stokes equations (Ahmed and Sunada 1969).

248 In the present study, the PSO algorithm was used to optimize the coefficients of the binomial

249 equation $(a, b)$. The values of the mentioned coefficients as well as the mean relative error 250 (MRE) between the computational and observational hydraulic gradients for all three types of 251 gradation (small, medium and large) are shown in Table 2.

252 Table 2. Optimized values of coefficients $a$ and $b$ and mean relative error (MRE) of hydraulic 253 gradient in steady flow condition

\begin{tabular}{|c|c|c|c|}
\hline Materials & $\mathbf{a}(\mathrm{s} / \mathrm{m})$ & $b\left(s^{2} / m^{2}\right)$ & MRE \% \\
\hline Small & 10.584 & 64.043 & 1.913 \\
\hline Medium & 7.981 & 13.705 & 3.614 \\
\hline Large & 5.965 & 8.635 & 3.322 \\
\hline
\end{tabular}

(Shokri et al. 2012; Hannoura and McCorquodale 1985) indicated that the third term of the

256 trinomial equation (Eq. 9) is negligible in calculation of the hydraulic gradient in the free

257 surface porous media. Hosseini and Joy (2007) used the coefficients of the binomial equation 
258 in steady flow condition to study the hydraulic gradient and solve the Saint-Venant equations

259 in the unsteady flow condition in porous media. The experimental results of the present study,

260 which were obtained using a confined porous media (steel cylinder), indicated the high

261 accuracy of the trinomial equation in comparison to the binomial equation in estimation of

262 the hydraulic gradient changes in terms of unsteady flow velocity. Table 3 shows the values

263 of the coefficients $a$ and $b$ of the binomial equation and the coefficients $a, b, c$ of the trinomial

264 equation when the values of the hydraulic gradient relative to the unsteady flow velocity are

265 used to optimize the coefficients. In addition, the values of the mean relative error (MRE)

266 between the computational and observational hydraulic gradients in the unsteady flow

267 condition using the coefficients $\mathrm{a}$ and $\mathrm{b}$ obtained from the steady flow condition (as

268 recommended by Hosseini and Joy 2007), coefficients a and b obtained from the unsteady

269 flow condition by assuming an insignificant value of coefficient $\mathrm{c}$ in the trinomial equation

270 (as recommended by Hannoura and McCorquodale 1985; Shokri et al. 2012) and by using

271 coefficients $\mathrm{a}, \mathrm{b}, \mathrm{c}$ and trinomial equation are shown in Table 3 . It is worth noting that the

272 mentioned coefficients were optimized using the PSO algorithm.

273

274

275

276

277

278

279 
Table 3. Values of the optimized coefficients a and $b$ using steady and unsteady flow data and coefficients $a, b, c$ of unsteady flow data and mean relative error (MRE) values of hydraulic gradient of unsteady flow

283

\begin{tabular}{cccccc}
\hline Regime & Equations & Coefficients & Small & Medium & Large \\
\hline \multirow{2}{*}{ Steady } & \multirow{2}{*}{ Binomial } & $\mathrm{b}\left(\mathrm{s}^{2} / \mathrm{m}^{2}\right)$ & 64.043 & 13.705 & 8.635 \\
& & $\mathrm{MRE} \%$ & 25.567 & 25.923 & 21.306 \\
\hline \multirow{2}{*}{ Unsteady } & \multirow{2}{*}{ Binomial } & $\mathrm{a}(\mathrm{s} / \mathrm{m})$ & 3.848 & 5.255 & 5.411 \\
& & $\mathrm{~b}\left(\mathrm{~s}^{2} / \mathrm{m}^{2}\right)$ & 331.714 & 165.084 & 80.344 \\
& & $\mathrm{MRE} \%$ & 10.024 & 22.599 & 12.941 \\
\hline \multirow{3}{*}{ Unsteady } & \multirow{3}{*}{ Trinomial } & $\mathrm{a}\left(\mathrm{s} / \mathrm{s}^{2} / \mathrm{m}^{2}\right)$ & 2.859 & 2.225 & 3.704 \\
& & $\mathrm{c}\left(\mathrm{s}^{2} / \mathrm{m}\right)$ & 10.369 & 51.369 & 14.582 \\
& & $\mathrm{MRE} \%$ & 7.806 & 14.106 & 10.506 \\
\hline
\end{tabular}

284 As can be seen from Table 3, if the coefficients $a$ and $b$ obtained from the steady flow condition were used to calculate the changes in the hydraulic gradient relative to the unsteady flow velocity, the mean relative error (MRE) values of 25.567, 25.923, and 21.306 were obtained for the small, medium and large grained materials, respectively. In the case of using the coefficients $a$ and $b$ obtained from the unsteady flow data, the corresponding MRE values of $10.024,22.599$ and $12.941 \%$, were obtained, respectively. In addition, by using the optimized coefficients $\mathrm{a}, \mathrm{b}$, and $\mathrm{c}$ of the trinomial equation, the corresponding MRE values of 7.806, 14.106 and $10.506 \%$ were obtained, respectively. The MRE values of the trinomial equation in comparison to those of the binomial equation in case of using the coefficients a and $b$ obtained from the unsteady flow condition for three gradation types improved by 22,38 and $19 \%$, respectively. The corresponding values in case of using the coefficients a and $b$ obtained from the steady flow condition improved by 70,46 and $51 \%$, respectively. In other

296 words, in order to calculate the hydraulic gradient in the unsteady flow condition, the use of 297 the coefficients $\mathrm{a}, \mathrm{b}$, and $\mathrm{c}$ of the unsteady flow and the trinomial equation, coefficients a and 
298 b obtained from the unsteady flow data, and coefficients a and b obtained from the steady

299 flow data were more accurate, respectively.

\section{$300 \quad 3.2$ Drag coefficient in porous media}

301 In addition to power equations (Eq. 2) and Forchheimer binomial equation (Eq. 3), one of the 302 methods of flow analysis in porous media is the use of friction coefficient (f) in terms of

303 Reynolds number (Re). Accordingly, the flow energy loss in porous media is obtained using

304 an equation similar to the Darcy-Weisbach equation (Stephenson 1979; Herrera and Felton 305 1991). For the confined flow in the pipes, the Darcy-Wiesbach equation is expressed using 306 Eq. (13).

$307 \quad h_{f}=f \frac{L}{d} \cdot \frac{V^{2}}{2 g}$

308 Where $\mathrm{f}$ is the (dimensionless) friction coefficient, $\mathrm{L}$ is the pipe length $(\mathrm{m}), \mathrm{d}$ is the pipe 309 diameter $(\mathrm{m}), \mathrm{V}$ is flow velocity $(\mathrm{m} / \mathrm{s}), \mathrm{g}$ is the acceleration of gravity $\left(\mathrm{m} / \mathrm{s}^{2}\right)$, and $\mathrm{h}_{\mathrm{f}}$ is the 310 energy loss (m).

311 Since $\mathrm{h}_{\mathrm{f}} / \mathrm{L}$ is equal to the hydraulic gradient, Eq. (13) can be rewritten as Eq. (14).

$312 \quad i=\frac{f}{d} \cdot \frac{V^{2}}{2 g}$

313 Leps (1973) indicated the velocity corresponding head in the above equations as $\frac{V^{2}}{m g}$, with $\mathrm{m}$

314 value of 2 according to the Darcy-Wiesbach equation in pipes. Stephenson (1979) assumed

315 the formation of the porous media inside a set of winding tubes within a set of aggregates

316 with pores, considered the value of $\mathrm{m}$ in the energy loss $\left(\mathrm{h}_{\mathrm{f}}\right)$ equation equal to 1 and proposed

317 Eq. (15) to calculate the hydraulic gradient.

$318 \quad i=\frac{f}{d} \cdot \frac{V^{2}}{g}$ 
319 According to the equations presented in fluid mechanics, Eq. (16) is presented to calculate the

320 friction force applied to the assumed control volume of porous media materials (Streeter 321 1962).

$322 F_{f}=\gamma A h_{f}$

323 The drag force is also expressed using Eq. (17) (Batchelor and Batchelor 2000).

$324 \quad F_{d}=C_{d} \gamma A \frac{V^{2}}{2 g}$

325 The external friction force is equal to the drag force. In other words, by equating Eqs. (16) 326 and (17), Eq. (18) was obtained.

$327 h_{f}=C_{d} \frac{V^{2}}{2 g}$

328 Where $\mathrm{V}$ is the flow velocity $(\mathrm{m} / \mathrm{s}), \mathrm{g}$ is the acceleration of gravity $\left(\mathrm{m} / \mathrm{s}^{2}\right)$, and $\mathrm{C}_{\mathrm{d}}$ is the drag 329 coefficient. By performing several experiments, (Haider and Levenspiel 1989; Swamee and 330 Ojha 1991; Ganser 1993; Chien 1994; Cheng 1997) proposed empirical equations for 331 calculation of the drag coefficient.

332 To calculate the energy loss $\left(\mathrm{h}_{\mathrm{f}}\right)$, by equating the Darcy-Wiesbach equation (Eq. 13) and the 333 equation obtained from drag force (Eq. 18), Eq. (19) was obtained.

$334 \quad C_{d}=f \frac{L}{d}$

335 Equation (19) indicates that for a given value of the friction coefficient (f), the value of the 336 drag coefficient $\left(\mathrm{C}_{\mathrm{d}}\right)$ and consequently, the drag force $\left(\mathrm{F}_{\mathrm{d}}\right)$ can be calculated. In order to 337 calculate $\mathrm{f}$ in terms of Reynolds number in porous media, Eq. (20) was proposed by Ahmed 338 and Sunada using the Navier-Stokes equations in steady flow condition (Ahmed and Sunada, 339 1969). 
$340 \quad f=\frac{1}{\operatorname{Re}}+1, \quad \operatorname{Re}=\frac{\rho V d}{\mu}$

341 Where $\rho$ is water density $(1000 \mathrm{~kg} / \mathrm{m} 3)$ and $\mu$ is water viscosity $(0.001 \mathrm{~kg} / \mathrm{m} . \mathrm{s})$. According

342 to Eqs. (19) and (20), the drag coefficient in the free surface porous media is calculated using

343 Eq. (21).

$344 \quad C_{d}=\left(\frac{1}{\operatorname{Re}}+1\right) \frac{L}{d}$

345 Where $\mathrm{d}$ is calculated using the equations proposed by Ahmed and Sunada (1969) 346 (Eq. 7, $\mathrm{K}=\mathrm{cd}^{2}$ ).

347 Results of the present study showed that the friction coefficient (f) in one-dimensional 348 confined porous media in the steady and unsteady flow conditions is different from Eq. (20).

349 3.3 Drag coefficient of steady flow in porous media

350 According to Darcy-Wiesbach equations (Eq. 15), the binomial equation (Eq. 3), and the 351 equation of coefficients $\mathrm{a}$ and $\mathrm{b}$ presented by Ahmed and Sunada (Eq. 7), the friction 352 coefficient (f) in one-dimensional confined porous media in the steady flow condition is 353 calculated using Eq. (22).

$354 f=\frac{2 i d g}{V^{2}}=\frac{2\left(\frac{\mu}{\rho g K} V+\frac{1}{g \sqrt{C K}} V^{2}\right) d g}{V^{2}}$

355 With simplification, Eq. (24) can be written as Eq. (23).

356

$f=\frac{2}{C}\left(\frac{\mu}{\rho V d}+1\right)=\frac{2}{C}\left(\frac{1}{\operatorname{Re}}+1\right)$

357 In other words, in the steady flow condition in one-dimensional confined porous media, the 358 friction coefficient ( $\mathrm{f}$ ) is $2 / \mathrm{C}$ times the friction coefficient ( $\mathrm{f}$ ) in the free surface porous media. 
359 The present study considering the steady flow condition in one-dimensional confined porous

360 media included the following steps:

361 1) Using experimental data, hydraulic gradient (i) (Fig. 4) in terms of flow velocity (V), the

362 optimized coefficients a and b using the PSO algorithm (Table 2), coefficients $\mathrm{K}$, $\mathrm{C}$ and $\mathrm{d}$

363 using Eq. (7) were calculated for small, medium and large-grained particles and summarized

364 in Table (4).

365 2) The friction coefficient (f) using Eq. (15), which is the same as the observational friction

366 coefficient, Reynolds number (Re) using Eq. (20), computational friction coefficient (f) using

367 the presented equation in the present study (Eq. 23) was calculated for the three gradations.

368 Figure 6 shows the changes in the friction coefficient (f) versus Reynolds number of steady

369 flow.

3703 3) Observational friction coefficient (calculated directly from experimental data and Eq. (15))

371 was compared with computational friction coefficient (calculated using Eq. (23), which was

372 presented in the present study for steady flow condition) and mean relative error (MRE)

373 values are shown in Table 4.

374 Table 4. Coefficients K, C, $d$ and mean relative error (MRE) values obtained from

375 observational and computational friction coefficients (f) in steady flow condition

\begin{tabular}{cccc}
\hline Coefficients & Small & Medium & Large \\
\hline K & $9.6 \mathrm{E}-9$ & $12.8 \mathrm{E}-9$ & $17.1 \mathrm{E}-9$ \\
$\mathbf{C}$ & 263.041 & 4331.704 & 8154.079 \\
$\mathbf{d}$ & $6.051 \mathrm{E}-6$ & $1.717 \mathrm{E}-6$ & $1.448 \mathrm{E}-6$ \\
MRE \% & 1.913 & 3.614 & 3.322 \\
\hline
\end{tabular}




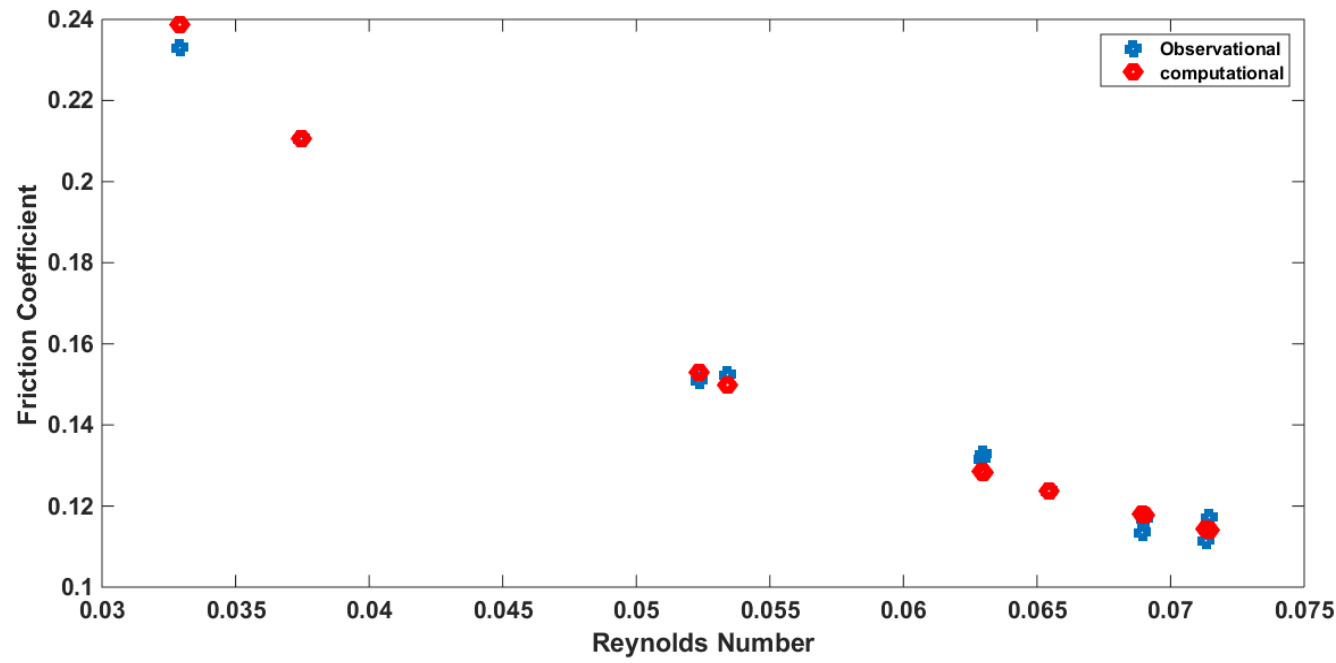

a. Small

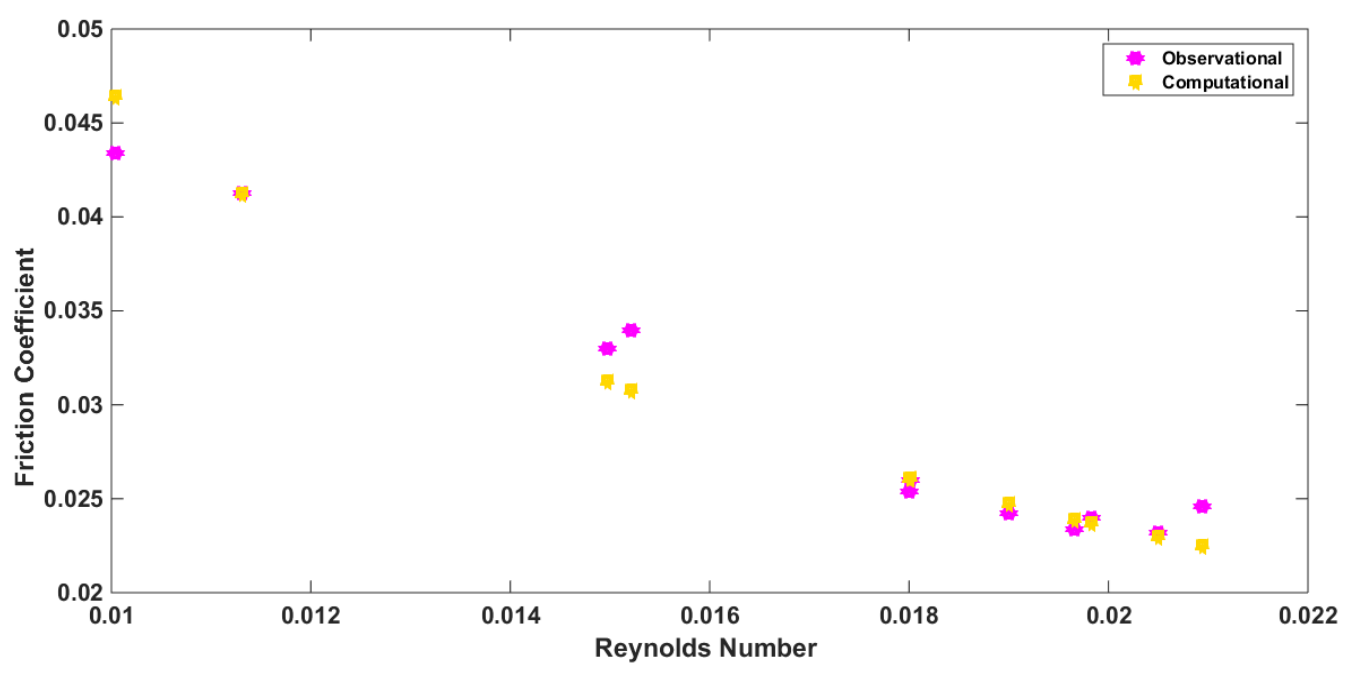

b. Medium

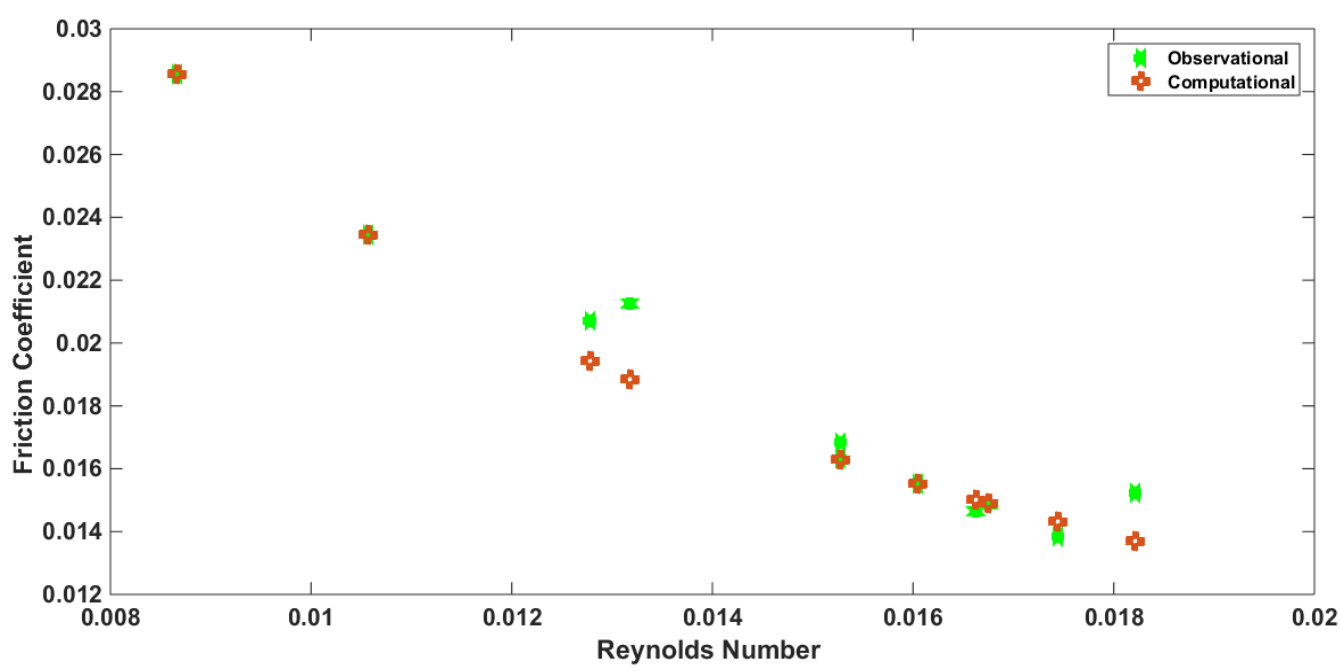

c. Large

383 Figure 6. Observational and computational friction coefficient versus Reynolds number in 
385 The mean relative error (MRE) values in Table 4 and Figure 6 indicated the high accuracy

386 and efficiency of the analytical equation proposed in the present study for calculation of the

387 coefficient $\mathrm{f}$ and consequently, $\mathrm{C}_{\mathrm{d}}$ and drag force $\left(\mathrm{F}_{\mathrm{d}}\right)$ in the steady flow condition in one-

388 dimensional confined porous media.

389 3.3 Drag coefficient of unsteady flow in porous media

390 According to Table 3, the trinomial equation (Eq. 9) is more accurate in estimation of the

391 hydraulic gradient of unsteady flow.

392 According to the Darcy-Wiesbach equations (Eq. 15), the trinomial equation (Eq. 9) and the 393 equation presented by Ahmad and Sunada (Eq. 7) for calculation of coefficients a and b, the

394 friction coefficient (f) in one-dimensional confined porous media in unsteady flow condition 395 is calculated using Eq. (24).

$396 f=\frac{2 i d g}{V^{2}}=\frac{2\left(\frac{\mu}{\rho g K} V+\frac{1}{g \sqrt{C K}} V^{2}+c \frac{d V}{d t}\right) d g}{V^{2}}$

397 By simplification, the coefficient $\mathrm{f}$ in confined porous media is obtained using Eq. (25).

398

$f=\frac{2}{C}\left(\frac{\mu}{\rho V d}+1\right)+\frac{2 d g}{V^{2}} \cdot c \frac{d v}{d t}=\frac{2}{C}\left(\frac{1}{\operatorname{Re}}+1\right)+\frac{2 d g}{V^{2}} \cdot c \frac{d v}{d t}$

399 Where $\mathrm{C}$ is the coefficient $\mathrm{f}$ obtained from the Ahmad and Sunada equation (Eq. 7) and c is 400 the coefficient of the third term of the trinomial equation (Eq. 9).

401 The calculation steps in the unsteady flow condition are the same as those for the steady flow 402 condition, with the difference that in the former, coefficients of the proposed trinomial 403 equation presented in Table 3 were used. In addition, to calculate the computational 404 coefficient $\mathrm{f}$ in terms of Re, the equation presented in the present study for unsteady flow (Eq. 25) was used. 
406 The values of the coefficients $\mathrm{K}, \mathrm{C}, \mathrm{d}$ and mean relative error (MRE) values between the 407 observational and computational friction coefficients (f) for three gradation types are shown

408 in Table 5. Changes in the observational and computational $\mathrm{f}$ in terms of Re are also shown in 409 Figure 7.

410 Table 5. Coefficients K, C, $d$ and mean relative error (MRE) values obtained from 411 observational and computational friction coefficients (f) in unsteady flow condition

\begin{tabular}{cccc}
\hline Coefficients & Small & Medium & Large \\
\hline K & $35.7 \mathrm{E}-9$ & $45.8 \mathrm{E}-9$ & $27.5 \mathrm{E}-9$ \\
$\mathbf{C}$ & 2.344 & 8.298 & 32.865 \\
$\mathbf{d}$ & $123 \mathrm{E}-6$ & $74.3 \mathrm{E}-6$ & $28.9 \mathrm{E}-6$ \\
MRE \% & 7.806 & 14.106 & 10.506
\end{tabular}

412

413

414

415

416

417

418

419 


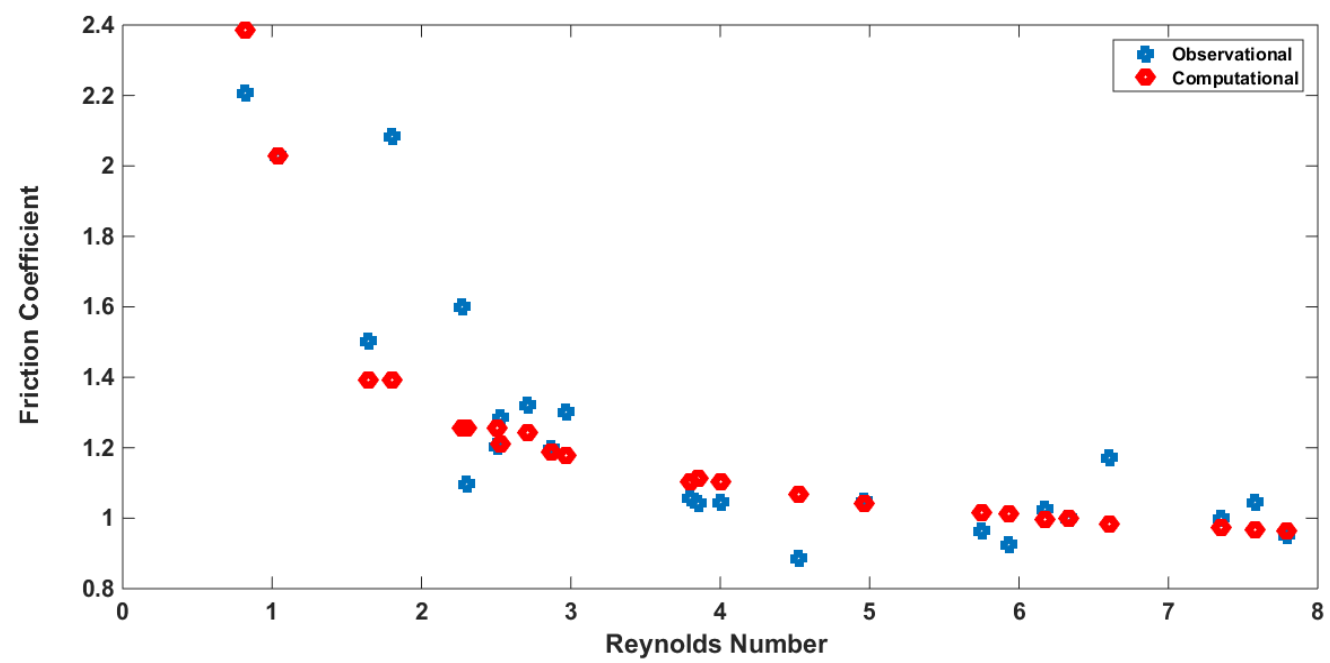

421

a. Small

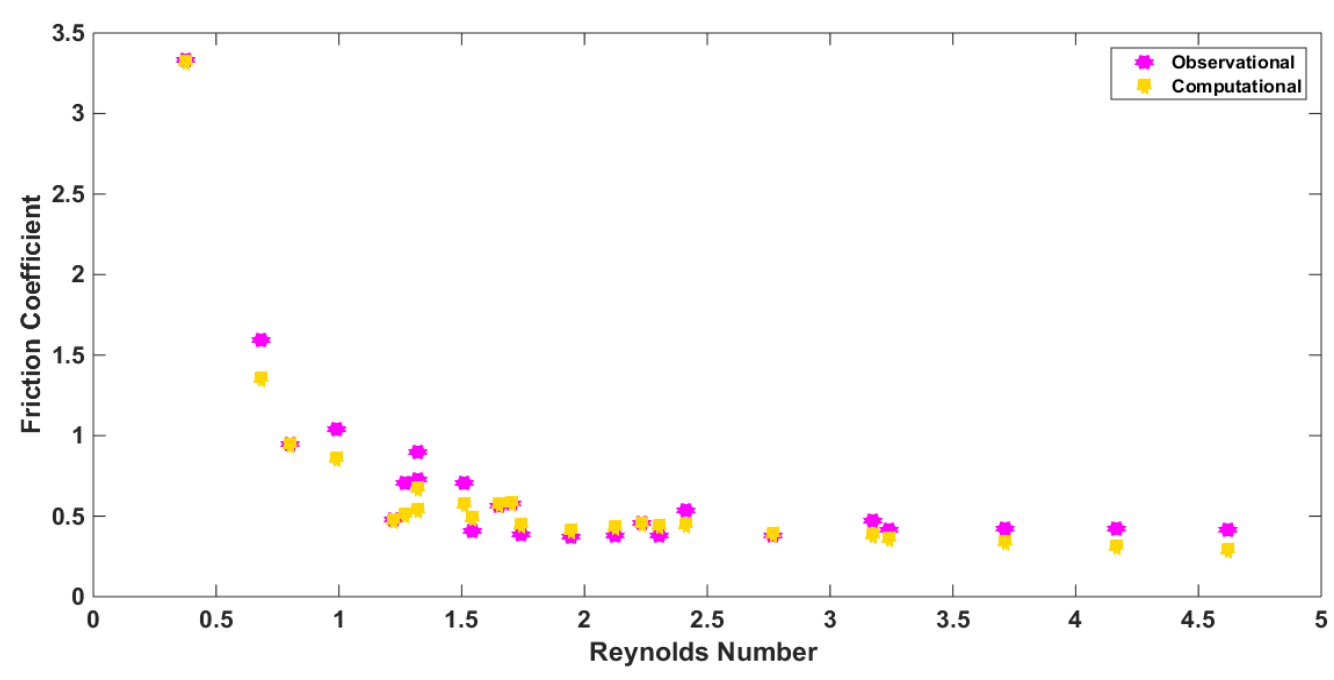

b. Medium

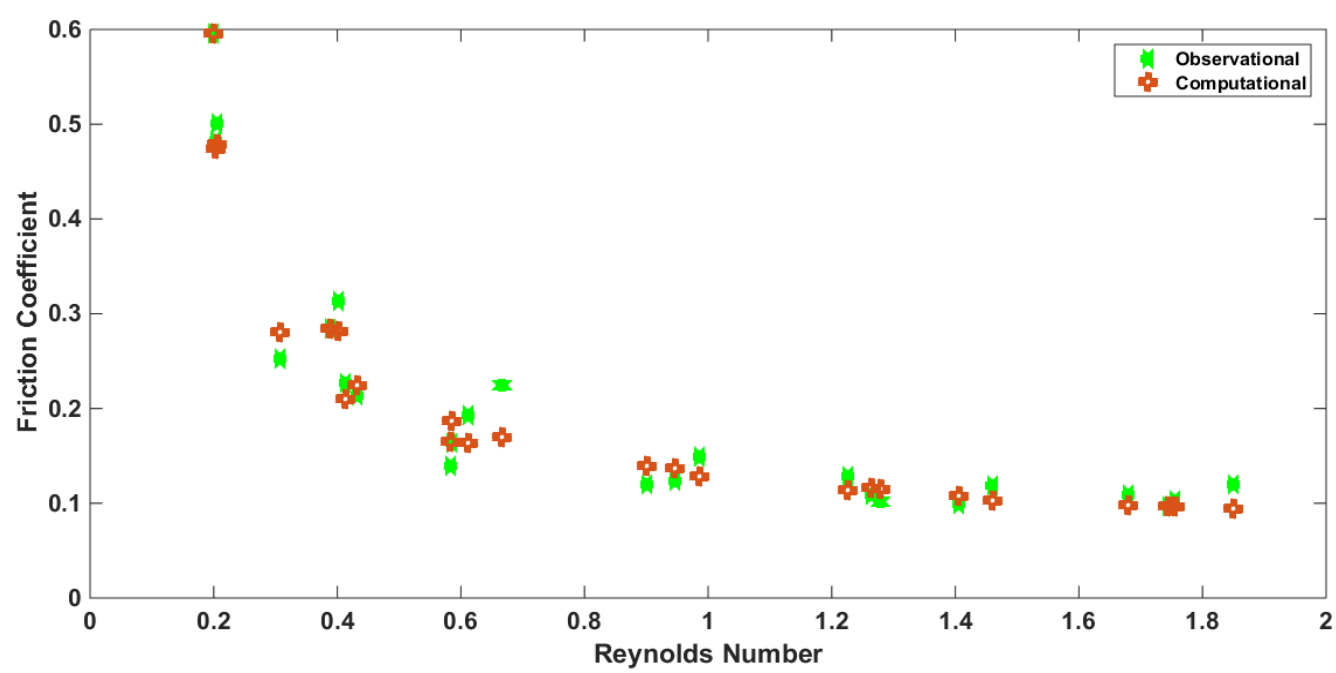

c. Large 
428 The mean relative error (MRE) values (Table 5) as well as the changes in the observational 429 and computational f values in terms of Re (Fig. 6) indicated the high accuracy and efficiency 430 of the proposed equation in the present study to calculate $\mathrm{f}$ and consequently, $\mathrm{C}_{\mathrm{d}}$ and $\mathrm{F}_{\mathrm{d}}$ for 431 unsteady flow in one-dimensional confined porous media.

\section{Conclusions}

433 In the present study, steady and unsteady flows in one-dimensional confined porous media 434 were studied to calculate the changes in hydraulic gradient (i) in terms of flow velocity (V) 435 and drag force $\left(\mathrm{F}_{\mathrm{d}}\right)$ considering small, medium and large grained materials. The calculation of 436 drag coefficient $\left(\mathrm{C}_{\mathrm{d}}\right)$ is the most important step in calculations of $F_{d}$. On the other hand, according to performed studies, the only important parameter in calculation of the $\mathrm{C}_{\mathrm{d}}$ in a porous media is the friction coefficient (f). For this reason, in the present study, to calculate the coefficient $\mathrm{f}$ in terms of Reynolds number (Re), the Darcy-Wiesbach equation in pipes,

440 the equations of $\mathrm{i}$ in terms of $\mathrm{V}$ (in the steady flow condition as a binomial equation and in 441 the unsteady flow condition as a trinomial equation), and the analytical equation by Ahmad and Sunada in calculation of the coefficients $a, b$ and coefficient $f$ in terms of Re in porous media, an equation for steady flow condition and another equation for unsteady flow

444 condition in one-dimensional confined porous media were presented, which have many 445 applications in civil engineering, mechanics, geology, and oil industry. The proposed 446 equations for the $\mathrm{f}$ coefficient in terms of $\mathrm{Re}$ in both steady and unsteady flow conditions 447 were evaluated using experimental data and the results showed the accuracy and efficiency of 448 these equations in estimation of the coefficient $\mathrm{f}$ and consequently, $\mathrm{C}_{\mathrm{d}}$ and $\mathrm{F}_{\mathrm{d}}$. In general, the 449 results of the present study include the followings.

450 1) Comparison of the accuracy of the hydraulic gradient calculations in terms of flow velocity 451 in unsteady flow conditions with the methods of a) trinomial equation, b) binomial equation 
and coefficients $\mathrm{a}$ and $\mathrm{b}$ obtained from unsteady flow data by assuming an insignificant coefficient $\mathrm{c}$, and C) binomial equation and coefficients $\mathrm{a}$ and $\mathrm{b}$ obtained from steady flow data showed that the methods a to $\mathrm{c}$ are more accurate, respectively.

2) Mean relative error (MRE) values between observational coefficients $f$ in the steady flow condition (direct use of experimental data to calculate coefficient $f$ ) and computational $f$ coefficients (using the proposed equation in the present study to calculate coefficient $\mathrm{f}$ in the steady flow condition) for small, medium and large grained materials were equal to 1.913, 3.614 and $3.322 \%$, respectively.

3) By using the proposed equation in the present study to calculate the coefficient $f$ in unsteady flow condition and then, comparing the computational and observational $\mathrm{f}$ values, the mean relative error (MRE) values of $7.806,14.106$ and $10.506 \%$ were obtained for the small, medium and large grained materials, respectively.

464 In other words, the results indicated that trinomial equation is more accurate in calculation of the hydraulic gradient changes with respect to the unsteady flow velocity. In addition, to calculate the coefficient $\mathrm{f}$ in terms of Re and consequently, the coefficient $\mathrm{C}_{d}$ in the porous media, instead of using the experimental and numerical equations obtained for a certain number of particles in the laboratory, the proposed equations in the present study for the steady and unsteady flow conditions with any number of particles and for all Reynolds numbers are suitably accurate and efficient.

471 Funding: The authors did not receive support from any organization for the submitted work.

472 Conflict of Interest: The authors have no financial or proprietary interests in any material 473 discussed in this article.

474 Availability of data and material: The authors certify the data and materials contained in 475 the manuscript. 
476 Code availability: The relationships used are presented in the manuscript and the description

477 of the particle swarm optimization (PSO) algorithm is also attached.

478 Authors' contributions: All authors have contributed to various sections of the manuscript.

479 Ethics approval: The authors heeded all of the Ethical Approval cases.

480 Consent to participate: The authors have studied the cases of the Authorship principles

481 section and it is accepted.

482 Consent for publication: The authors certify the policy and the copyright of the publication.

483 All authors contributed to the study conception and design. Material preparation, data 484 collection and analysis were performed by [Hadi Norouzi], [Jalal Bazargan], [Faezeh 485 Azhang] and [Rana Nasiri]. The first draft of the manuscript was written by [Hadi Norouzi] 486 and all authors commented on previous versions of the manuscript. All authors read and 487 approved the final manuscript.

\section{$488 \quad 5$ References}

489 Abbas, W., Awadalla, R., Bicher, S., Abdeen, M. A., \& El Shinnawy, E. S. M. (2021). Semi-analytical solution 490 of nonlinear dynamic behaviour for fully saturated porous media. European Journal of Environmental and Civil 491 Engineering, 25(2), 264-280.

492 Afshar, A., Kazemi, H., \& Saadatpour, M. (2011). Particle swarm optimization for automatic calibration of large 493 scale water quality model (CE-QUAL-W2): Application to Karkheh Reservoir, Iran. Water resources 494 management, 25(10), 2613-2632.

495 Ahmed, N. and Sunada, D. K. 1969. Nonlinear flow in porous media. Journal of the Hydraulics Division, 95(6): $496 \quad 1847-1858$.

497 Bari, R. and Hansen, D. (2002). Application of gradually-varied flow algorithms to simulate buried 498 streams. Journal of Hydraulic Research. 40(6): 673-683.

499 Batchelor, C. K., \& Batchelor, G. K. (2000). An introduction to fluid dynamics. Cambridge university press. 
500 Bazargan, J., Norouzi, H. (2018). Investigation the Effect of Using Variable Values for the Parameters of the 501 Linear Muskingum Method Using the Particle Swarm Algorithm (PSO). Water Resources Management, 32(14), $502 \quad 4763-4777$

503 Bazargan, J., Shoaei, S. M., (2006). Discussion, "Application of gradually varied flow algorithms to simulate buried streams." IAHR J. of Hydraulic Research, Vol 44, No 1, 138-141.

Bechler, A., Romary, T., Jeannée, N., \& Desnoyers, Y. (2013). Geostatistical sampling optimization of contaminated facilities. Stochastic environmental research and risk assessment, 27(8), 1967-1974.

507

Bird RB, \& Stewart WE, Lightfoot EN. (2007). Transport phenomena. Second ed. London: John Wiley and 508 Sons.

509 Cao, K., \& Ye, X. (2013). Coarse-grained parallel genetic algorithm applied to a vector based land use

510 allocation optimization problem: the case study of Tongzhou Newtown, Beijing, China. Stochastic 511 Environmental Research and Risk Assessment, 27(5), 1133-1142.

512 Chau, K. (2005). A split-step PSO algorithm in prediction of water quality pollution. In International 513 Symposium on Neural Networks (pp. 1034-1039). Springer, Berlin, Heidelberg.

514 Chau, K.W., (2007). A split-step particle swarm optimization algorithm in river stage forecasting. J. Hydrol. 34, $515 \quad 131-135$.

516 Cheng, N. S. (1997). Simplified settling velocity formula for sediment particle. Journal of hydraulic 517 engineering, 123(2), 149-152.

518 Chien, S. F. (1994). Settling velocity of irregularly shaped particles. SPE Drilling \& Completion, 9(04), 281519289.

520 Chen, R. C., \& Wu, J. L. (2000). The flow characteristics between two interactive spheres. Chemical engineering science, 55(6), 1143-1158.

522 Chu, H. J., Chang, L. C. (2009). Applying particle swarm optimization to parameter estimation of the nonlinear 523 Muskingum model. Journal of Hydrologic Engineering, 14(9), 1024-1027.

524 Clerc, M., Kennedy, J., (2002). The particle swarm-explosion, stability, and convergence in a multidimensional 525 complex space. IEEE Trans. Evol. Comput. 6 (1), 58-73. 
526 Di Felice, R. (1994). The voidage function for fluid-particle interaction systems. International Journal of 527 Multiphase Flow, 20(1), 153-159.

528 Di Cesare, N. Chamoret, D. and Domaszewski, M. (2015). A new hybrid PSO algorithm based on a stochastic 529 Markov chain model. Advances in Engineering Software. 90: 127-137.

530 Eberhart, R. and Kennedy, J. (1995). A new optimizer using particle swarm theory. In MHS'95. Proceedings of 531 the Sixth International Symposium on Micro Machine and Human Science (pp. 39-43). IEEE.

532 Ergun, S. (1952). Fluid Flow through Packed Columns. Chemical Engineering Progress. 48: 89-94.

533 Fand, R. M., \& Thinakaran, R. (1990). The influence of the wall on flow through pipes packed with spheres.

534 Forchheimer, P. (1901). Wasserbewagung Drunch Boden, Z.Ver, Deutsh. Ing. 45: 1782-1788.

535 Ganser, G. H. (1993). A rational approach to drag prediction of spherical and nonspherical particles. Powder 536 technology, 77(2), 143-152.

537 Gudarzi, M., Bazargan, J., Shoaei, S. (2020). Longitude Profile Analysis of Water Table in Rockfill Materials 538 Using Gradually Varied Flow Theory with Consideration of Drag Force. Iranian Journal of Soil and Water 539 Research, 51(2), 403-415. doi: 10.22059/ijswr.2019.287292.668295

540 Gurarslan, G., Karahan, H., (2011). Parameter Estimation Technique for the Nonlinear Muskingum Flood 541 Routing Model. In: 6thEWRA International Symposium-Water Engineering and Management in a Changing 542 Environment, Catania, Italy.

543 Hannoura, A. A., McCorquodale, J. A., (1985). Rubble Mounds: Hydraulic Conductivity Equation, J.

544 Waterway, Port, Costal and Ocean Engineering, ASCE, 111(5), 783-799.

545 Hansen, D. Garga, V. K. and Townsend, D. R. (1995). Selection and application of a one-dimensional non-

546 Darcy flow equation for two-dimensional flow through rockfill embankments. Canadian Geotechnical 547 Journal. 32(2): 223-232.

548 Haider, A., \& Levenspiel, O. (1989). Drag coefficient and terminal velocity of spherical and nonspherical 549 particles. Powder technology, 58(1), 63-70.

550 Herrera, N. M., \& Felton, G. K. (1991). HYDRAULICS OF FLOW THROUGH A ROCKHLL DAM USING

551 SEDIMENT-FREE WATER. Transactions of the ASAE, 34(3), 871-0875. 
Hill, R. J., Koch, D. L., \& Ladd, A. J. (2001a). The first effects of fluid inertia on flows in ordered and random arrays of spheres. Journal of Fluid Mechanics, 448, 213.

Hill, R. J., Koch, D. L., \& Ladd, A. J. (2001b). Moderate-Reynolds-number flows in ordered and random arrays of spheres. Journal of Fluid Mechanics, 448, 243.

Hoang, H., Hoxha, D., Belayachi, N., \& Do, D. P. (2013). Modelling of two-phase flow in capillary porous medium by a microscopic discrete approach. European journal of environmental and civil engineering, 17(6), $444-452$.

Hsu, K. C., \& Chen, K. C. (2010). Multiscale flow and transport model in three-dimensional fractal porous media. Stochastic Environmental Research and Risk Assessment, 24(7), 1053-1065. approximation simulated annealing optimization algorithm for parameters calibration of porous media flow model. Stochastic Environmental Research and Risk Assessment, 33(2), 395-406.

565 Jiang, X., Lu, W., Na, J., Hou, Z., Wang, Y., \& Chi, B. (2018). A stochastic optimization model based on 566 adaptive feedback correction process and surrogate model uncertainty for DNAPL-contaminated groundwater 567 remediation design. Stochastic Environmental Research and Risk Assessment, 32(11), 3195-3206.

568 Karahan, H., (2012). Determining rainfall-intensity-duration-frequency relationship using Particle Swarm

569 Optimization. KSCE J. Civil Eng. 16 (4), 667-675.

570 Kumar, D.N., Reddy, M.J., (2007). Multipurpose reservoir operation using particle swarm optimization. J. 571 Water Resour. Plan. Manage. ASCE 133 (3), 192-201.

572 Kovács, G. (1980). Developments in water science: seepage hydraulics. Elsevier Scientific Publishing 573 Company.

574 Lei, T., Meng, X., \& Guo, Z. (2017). Pore-scale study on reactive mixing of miscible solutions with viscous 575 fingering in porous media. Computers \& Fluids, 155, 146-160.

576 Leps, T. M. (1973). Flow through rockfill, Embankment-dam engineering casagrande volume edited by 577 Hirschfeld, RC and Poulos, SJ. 
578 Liang, S. C., Hong, T., \& Fan, L. S. (1996). Effects of particle arrangements on the drag force of a particle in the 579 intermediate flow regime. International journal of multiphase flow, 22(2), 285-306.

580 Lu, W. Z., Fan, H. Y., Leung, A. Y. T., \& Wong, J. C. K. (2002). Analysis of pollutant levels in central Hong 581 Kong applying neural network method with particle swarm optimization. Environmental monitoring and 582 assessment, 79(3), 217-230.

583 Mccorquodale, J. A., Hannoura, A. A. A., Sam Nasser, M. (1978). Hydraulic conductivity of rockfill. Journal of 584 Hydraulic Research, 16(2), 123-137.

585 Moghaddam, A., Behmanesh, J., Farsijani, A. (2016). Parameters estimation for the new four-parameter 586 nonlinear Muskingum model using the particle swarm optimization. Water resources management, 30(7), 21435872160.

588 Nagesh Kumar, D., Janga Reddy M. (2007). Multipurpose reservoir operation using particle swarm optimization. J Water Resour Plan Manag 133:192-201.

590 Norouzi, H., Bazargan, J. (2020). Flood routing by linear Muskingum method using two basic floods data using 591 particle swarm optimization (PSO) algorithm. Water Supply.

592 Norouzi, H., \& Bazargan, J. (2021). Effects of uncertainty in determining the parameters of the linear 593 Muskingum method using the particle swarm optimization (PSO) algorithm. Journal of Water and Climate 594 Change.

595 Rahimi, M., Schoener, Z., Zhu, X., Zhang, F., Gorski, C. A., \& Logan, B. E. (2017). Removal of copper from 596 water using a thermally regenerative electrodeposition battery. Journal of hazardous materials, 322, 551-556.

597 Rong, L. W., Dong, K. J., \& Yu, A. B. (2013). Lattice-Boltzmann simulation of fluid flow through packed beds 598 of uniform spheres: Effect of porosity. Chemical Engineering Science, 99, 44-58.

599 Schlichting, H., Gersten, K. (2000). Boundary layer theory, eighth ed. Springer Verlag, Berlin.

600 Sedghi-Asl, M., Rahimi, H. (2011). Adoption of Manning's equation to 1D non-Darcy flow problems. Journal of 601 Hydraulic Research, 49(6), 814-817.

602 Stephenson, D. J. (1979). Rockfill in hydraulic engineering. Elsevier scientific publishing compani. Distributors 603 for the United States and Canada. 
604 Sidiropoulou, M. G. Moutsopoulos, K. N. Tsihrintzis, V. A. (2007). Determination of Forchheimer equation

605 coefficients a and b. Hydrological Processes: An International Journal. 21(4): 534-554.

606 Sheikh, B., \& Pak, A. (2015). Numerical investigation of the effects of porosity and tortuosity on soil

607 permeability using coupled three-dimensional discrete-element method and lattice Boltzmann method. Physical

608 Review E, 91(5), 053301.

609 Sheikh, B., \& Qiu, T. (2018). Pore-scale simulation and statistical investigation of velocity and drag force

610 distribution of flow through randomly-packed porous media under low and intermediate Reynolds

611 numbers. Computers \& Fluids, 171, 15-28.

612 Shi, Y. and Eberhart, R. (1998). A modified particle swarm optimizer. In 1998 IEEE international conference on

613 evolutionary computation proceedings. IEEE world congress on computational intelligence (Cat. No.

$61498 T H 8360)($ pp. 69-73). IEEE.

615 Shokri, M., Saboor, M., Bayat, H., Sadeghian, J. (2012). Experimental Investigation on Nonlinear Analysis of

616 Unsteady Flow through Coarse Porous Media. Journal of Water and Wastewater; Ab va Fazilab (in persian),

617 23(4), 106-115.

618 Song, Z., Li, Z., Wei, M., Lai, F., \& Bai, B. (2014). Sensitivity analysis of water-alternating-CO2 flooding for

619 enhanced oil recovery in high water cut oil reservoirs. Computers \& Fluids, 99, 93-103.

620 Streeter, V. L. (1962). Fluid mechanics, McCraw-Hill Book Company.

621 Swamee, P. K., \& Ojha, C. S. P. (1991). Drag coefficient and fall velocity of nonspherical particles. Journal of 622 Hydraulic Engineering, 117(5), 660-667.

623 Van der Hoef, M. A., Beetstra, R., \& Kuipers, J. A. M. (2005). Lattice-Boltzmann simulations of low-Reynolds624 number flow past mono-and bidisperse arrays of spheres: results for the permeability and drag force. Journal of 625 fluid mechanics, 528, 233.

626 Ward, J. C. (1964). Turbulent flow in porous media. Journal of the hydraulics division. 90(5): 1-12.

627 Wen, C. Y., \& Yu, Y. H. (1966). A generalized method for predicting the minimum fluidization 628 velocity. AIChE Journal, 12(3), 610-612. 
629 Yin, X., \& Sundaresan, S. (2009). Fluid-particle drag in low-Reynolds-number polydisperse gas-solid 630 suspensions. AIChE journal, 55(6), 1352-1368.

631 Zhang, Y., Ge, W., Wang, X., \& Yang, C. (2011). Validation of EMMS-based drag model using lattice 632 Boltzmann simulations on GPUs. Particuology, 9(4), 365-373.

633 Zhang, T., Du, Y., Huang, T., Yang, J., Lu, F., \& Li, X. (2016). Reconstruction of porous media using 634 ISOMAP-based MPS. Stochastic environmental research and risk assessment, 30(1), 395-412.

635 Zhu, C., Liang, S. C., \& Fan, L. S. (1994). Particle wake effects on the drag force of an interactive 636 particle. International journal of multiphase flow, 20(1), 117-129.

637 Zhu, X., Rahimi, M., Gorski, C. A., \& Logan, B. (2016). A thermally-regenerative ammonia-based flow battery 638 for electrical energy recovery from waste heat. ChemSusChem, 9(8), 873-879. 


\section{Particle Swarm Optimization Algorithm}

This algorithm was first introduced by Eberhart and Kennedy in 1995 (Eberhart and Kennedy, 1995). The Particle Swarm Optimization Algorithm is a population-based search algorithm such as genetic algorithm, ant colony algorithm, bee algorithm, etc. It is a nature-inspired algorithm designed based on collective intelligence and social behavior of birds and fish (Abido, 2002). The advantages of the Particle Swarm Optimization Algorithm include simple structure and implementation, low number of controllable parameters and high convergence speed as well as high computational efficiency (Abido, 2002; Del Valle et al. 2008). The structure of this algorithm will be discussed below.

\subsection{Initial population creation}

This algorithm starts by generating a random number of particles. Each of these particles is a possible answer to the optimization problem. Increasing the number of particles makes the algorithm complex. Of course, this increase in the initial population reduces the number of iterations of the algorithm. There must be a compromise between these two parameters. The number of this initial population as well as the number of iterations of the algorithm depends on the type and nature of the optimization problem.

In Particle Swarm Optimization Algorithm, each particle i has a position vector and a velocity vector, which are defined as equations (1) and (2) (Clerc, 2010).

$x_{i}=\left[x_{i 1}, x_{i 2}, \ldots, x_{i n}\right]$

$v_{i}=\left[v_{i 1}, v_{i 2}, \ldots, v_{i n}\right]$ 
$x_{i}$ : The current location of the $i_{\text {th }}$ particle

$v_{i}:$ The current speed of the $\mathrm{i}_{\text {th }}$ particle

$\mathrm{n}$ in the above equations is the dimension of the search space of the optimization problem.

The Particle Swarm Optimization Algorithm must consider a variable that holds the best position of each particle in its memory. This variable is considered as $x^{i B e s t}$. Where $\mathrm{i}$ is considered to represent the number of the particle. In the other word, $x^{i \text { iBest }}$ is cost function having the lowest value (or the profit or fitness function having the highest value). In the next step, after generating a random initial population, the variables $x^{\text {iBest }}$ and $\mathrm{x}^{\text {gBest }}$ must be quantified according to equations 3 and 4 . In equation $3, x^{g B e s t}$ is the best particle among the community of particles. As $\mathrm{x}^{\text {gBest }}$ does not belong to any particular particle, index $\mathrm{i}$ has not been applied. As seen in equation 4, at this stage of the algorithm, because the particles have no motion, and are newly generated, $x^{i B e s t}$ is equal to $\mathrm{x}_{\mathrm{i}} \cdot x^{i B e s t}$ is the best personal experience of the $\mathrm{i}_{\text {th }}$ particle (Clerc, 2010).

$$
\begin{aligned}
& x^{g B e s t}(t+1)= \begin{cases}x_{i}(\mathrm{t}) & \operatorname{Cost}\left(x_{i}(\mathrm{t})\right)<\operatorname{Cost}\left(x^{g B e s t}\right) \\
x^{g B e s t} & \operatorname{Cost}\left(x_{i}(\mathrm{t})\right)>\operatorname{Cost}\left(x^{g B e s t}\right)\end{cases} \\
& x^{i B e s t}(\mathrm{t}+1)=x_{i}(\mathrm{t})
\end{aligned}
$$

\subsection{The particles movement toward the best particle}

At this stage of the algorithm, a movement should be considered for the particles generated in the previous section. Equation 3 is used to change the location of each particle. In this equation, two random functions of $r_{1}$ and $r_{2}$ with uniform distribution are used to model the stochastic 
nature of the algorithm. Speed update equation is in the form of equation 5 . In this equation, $\mathrm{r}_{1}$ and $\mathrm{r}_{2}$ are scaled using $\mathrm{c}_{1}$ and $\mathrm{c}_{2}$. In this equation, $0<\mathrm{c}_{1}, \mathrm{c}_{2}<2$, that these coefficients are known as Acceleration Coefficients. It is called so because if the value of equation 5 is rewritten as the equation 6 , by dividing the two sides of the equation 6 in unit of time, the value on the left of the equation represents the acceleration coefficients. The acceleration coefficients have an effect on each step of each particle in each reiteration. In the other word, the value of $\mathrm{c}_{1}$ represents the affectivity of a particle from its best memory position and the value of $c_{2}$ represents the affectivity of the particle from the total. In equations 5 and 6 , index $\mathrm{j}$ represents the $\mathrm{j}$ th dimension of each particle in which $\mathrm{j}$ is $\mathrm{j}-0,1, \ldots . . \mathrm{n}$ (Eberhart and Kennedy, 1995).

$$
\begin{aligned}
& v^{i}[\mathrm{t}+1]=\mathrm{v}^{i}[t]+c_{1} r_{1, j}\left(\mathrm{x}^{i B e s t}[\mathrm{t}]-\mathrm{x}^{i}[\mathrm{t}]\right)+c_{2} r_{2, j}\left(\mathrm{x}^{\text {gBest }}[\mathrm{t}]-\mathrm{x}^{i}[\mathrm{t}]\right) \\
& v^{i}[\mathrm{t}+1]-\mathrm{v}^{i}[t]=c_{1} r_{1, j}\left(\mathrm{x}^{i B e s t}[\mathrm{t}]-\mathrm{x}^{i}[\mathrm{t}]\right)+c_{2} r_{2, j}\left(\mathrm{x}^{\text {gBest }}[\mathrm{t}]-\mathrm{x}^{i}[\mathrm{t}]\right)
\end{aligned}
$$

In the above equation, over time, if a particle has cost function less (or benefit function higher than) the $\mathrm{x}^{\text {gBest }}$, it will replace this particle and the cost value and position of the particle will be updated. The speed update equation has three components. The first component of this equation corresponds to the velocity of the particle in the previous step and is therefore called the inertia component. This component reflects the tendency of the particle group to maintain its orientation in the search space. As shown in Equation (6), the performance of the algorithm is influenced by the best position of each particle, the best individual experience of the particle (the best individual experience of the particle) as well as the position of the best neighbor particle in the neighborhood of the same particle ( the best collective experience). Therefore, each particle with a special ratio will be attracted toward its best value and its best neighbor particle. Therefore, the 
second component of this equation is called the cognitive component and the third component is called the social component.

\subsection{Inertia coefficient}

The value of velocity vector $v^{i}[t]$ in equation (5) can be considered as a variable. This vector is weighted by which is called the inertia coefficient. This parameter is one of the important parameters in the Particle Swarm Optimization Algorithm that its proper tuning makes this algorithm robust (Ting et al. 2012). This parameter was first introduced by Shay and Eberhart in 1998 and added to the velocity equation (Shi and Eberhart, 1998). By incorporating this parameter into Equation (5), it is modified into Equation (6) (Di Cesare et al. 2015). To improve the convergence of the algorithm, the coefficient can be adjusted so that it decreases by passing time and approaching to the optimal response. Adjusting this parameter provides a variety of linear, nonlinear, and adaptive functions such as Constant inertia weight, Random inertia Weight, linear decreasing inertia weight, Oscillating Inertia Weight, etc. in reference (Bansal et al. 2011), the authors have discussed 15 of these functions and have investigated the performance of these functions on function 5, which is discussed in the following sections. The decreasing trend of the inertia coefficient and consequently the decrease of the inertia component of the velocity equation is due to the particle moving initially with larger steps and as approaching to the final answer to the optimization problem, decreasing the particles step makes the particles not to get far from the optimal response. This modification can be done by multiplying coefficient w into a damping constant, so that at the end of each iteration in the main circle of this algorithm, this constant is re-multiplied into w. Moreover, the value of inertia coefficient can be defined as equation (8) (Eberhart et al. 2001; Lee and Park, 2006). In this equation, $\omega_{\min }$ and $\omega_{\max }$, 
respectively, represent the initial and final values of inertia coefficient and Iter and Iter $_{\text {Max }}$ represent Current iteration number and iteration number.

$$
\begin{aligned}
& v^{i}[\mathrm{t}+1]=w \mathrm{v}^{i}[t]+c_{1} r_{1, j}\left(\mathrm{x}^{i \text { Best }}[\mathrm{t}]-\mathrm{x}^{i}[\mathrm{t}]\right)+c_{2} r_{2, j}\left(\mathrm{x}^{g \text { Best }}[\mathrm{t}]-\mathrm{x}^{i}[\mathrm{t}]\right) \\
& w=\omega_{\text {Max }}-\frac{\omega_{\text {Max }}-\omega_{\text {Min }}}{\text { Iter }_{\text {Max }}} \times \text { Iter }
\end{aligned}
$$

Given the neighborhood concept of each particle and the social intelligence of the Particle Swarm Optimization Algorithm or the third component of equation (7), topologies are presented as follows.

\subsection{Particle Swarm Evolutionary Algorithm Models}

In general, the Particle Swarm Optimization Algorithm is examined by two main models. 1) Global best model (Gbest) and Local best model (Lbest). The main difference between the two models is in structure of the neighborhood model of each particle. In the first model, the neighborhood of each particle contains all members of the population and only one particle is known as the best particle, and all the particles in the group are absorbed by it. In this model, the best particle information is shared with the rest of the particles. Unlike the Gbest model, in Lbest, each particle only has access to the information related to the neighborhood of the same particle. In Gbest model, because all particles of the group is absorbed by a single particle, this model has a higher convergence rate than the Lbest model. One on the other, the probability of this model being trapped at local extreme points is higher than in the case of several defined neighborhoods (Poli et al. 2007; Mavrovouniotis et al. 2017).

For these models several topologies are presented which will be explained in the next section. 


\subsection{Types of network topologies or structures}

\subsubsection{Definition of particle topology}

Particle topology is the symbolic network structure of particles that reflects how the population particles interact and share information with each other.

As mentioned in the previous sections, this algorithm can be defined as a set of particles moving in areas determined by the best successful experience of each particle and the best experience of some other particles. According to the phrase "best experience of some other particles", there are many structures in the references that three main cases will be discussed below.

1. Star

2. Ring (Circles)

3. Wheel

\subsubsection{Star structure}

In the star structure, all the particles in the group are adjacent to each other. Therefore, the position of the best particle of the group is shared with all the particles of the group and affects their velocity updating equation. This structure is related to the Gbest model. In this structure, the probability of being trapped at the optimal local point is increased if the best solution of the problem is not close to the best particle. The properties of this structure can be attributed to its rapid convergence as well as the greater likelihood of being trapped in optimal local locations. The star structure is shown in the figure 1. 


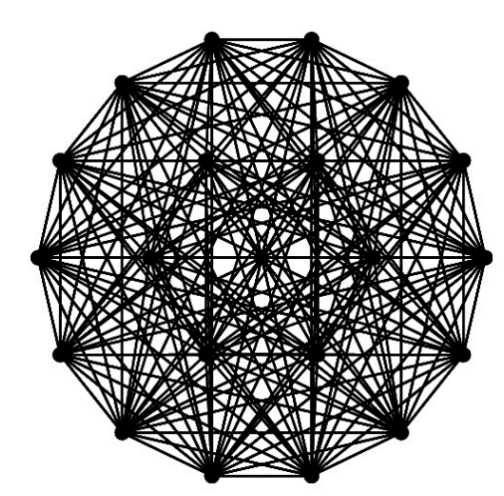

Fig. 1. Star structure

\subsubsection{Ring (Circles) structure}

In this structure, each particle is adjacent to its $n$ particle. So that there are $n / 2$ particles on each side. This structure for state $n=2$ is shown in Fig. (2). In this case, each particle is associated with its two adjacent particles. This structure is corresponding to the Lbest model. In this structure, each particle strives to move toward the best particle in its defined neighborhood. In this structure more areas of the search space are examined, but the convergence rate of this structure is low (Kacprzyk, 2009).

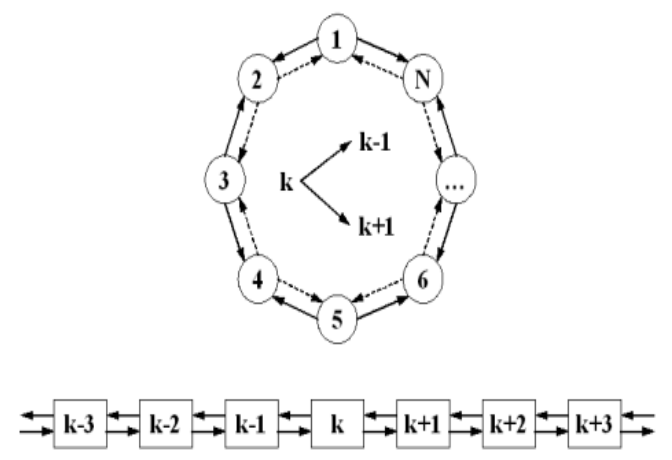

Fig. 2. Ring structure in $n=2$ 


\subsubsection{Wheel structure}

In the wheel structure, one particle is considered as the Focal particle (Hub). In this structure, the middle particle is attached to all the particles in the group, but the other particles are only attached to this particle and are insulated from each other. The focal particle moves to the best particle. If this focal particle movement improves its performance, it also affects other particles. This structure is shown in Fig. 3.

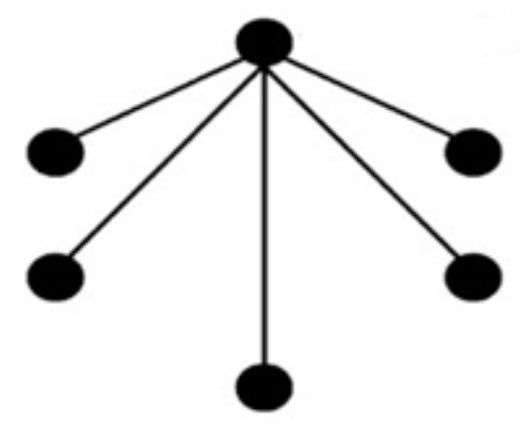

Fig. 3. Wheel structure

\subsection{Improved convergence of Particle Swarm Optimization Algorithm}

Morris Clarke and James Kennedy have proposed a method for selecting coefficients in equation (7) to improve the convergence of Particle Swarm Optimization Algorithms. In this method, equation (7) is modified in form of equation (9) (Clerc and Kennedy, 2002).

$v^{i}[\mathrm{t}+1]=\chi\left(\mathrm{v}^{i}[t]+c_{1} r_{1, j}\left(\mathrm{x}^{i B e s t}[\mathrm{t}]-\mathrm{x}^{i}[\mathrm{t}]\right)+c_{2} r_{2, j}\left(\mathrm{x}^{\text {gBest }}[\mathrm{t}]-\mathrm{x}^{i}[\mathrm{t}]\right)\right)$

In the recent equation, $\chi$ is Constriction coefficient which is defined as equation (10).

$\chi=\frac{2}{\left|2-\varphi-\sqrt{\varphi^{2}-4 \varphi}\right|}$ 
In the recent equation, coefficient $\varphi$ is defined as equation 11 (Chan et al. 2007).

$\varphi=c_{1}+c_{2} \quad \varphi>4$

\subsection{Limiting velocity}

After obtaining the particle velocity vector of the group, it is necessary to check whether the velocities obtained are within the specified permissible range. This allowed range is usually expressed as a coefficient of the width of the search space. This range is shown in Equations (12) and (13).

$v_{\text {max }}=\alpha\left(x_{\text {max }}-x_{\text {min }}\right)$

$v_{\text {min }}=-\alpha\left(x_{\text {max }}-x_{\text {min }}\right)$

In both recent equations, $x_{\min }$ and $x_{\max }$ represent the variables range in optimization problem. In addition, Alpha coefficient has a value between zeros to one, so that the velocity threshold value does not exceed the width of the research space.

After determining the particle velocity vector and determining the particle velocity threshold, these ranges are applied as relation (14):

$$
v^{i, j}(t+1)= \begin{cases}v_{\min } & v^{i, j}(t+1) \leq v^{j}{ }_{\text {min }} \\ v^{i, j}(t+1) & v_{\text {min }}^{j}<v^{i, j}(t+1)<v_{\text {max }}^{j} \\ v_{\text {max }} & v^{i, j}(t+1) \geq v^{i, j}{ }_{\text {max }}\end{cases}
$$


At this point, new values of group particle velocity are set and the necessary constraints are applied. Now the new position of the particles must be determined. This new position is determined by Equation (15).

$x(t+1)=x(t)+v(t+1)$

Displacement of the particle based on the velocity update equation and the displacement equation has been shown in Fig. (4). After the displacement of the particle following the mentioned steps, the above steps are repeated until the termination conditions of the algorithm are satisfied and the best particle position among all the group members is delivered as the optimal response.

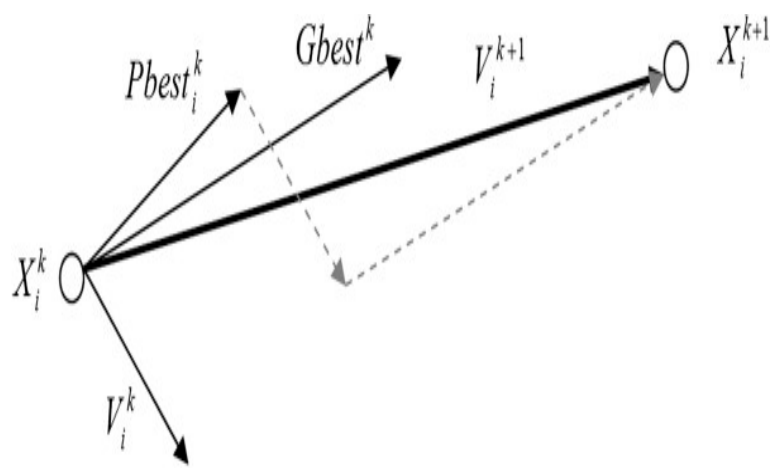

Fig. 4. Displacement of the $i_{\text {th }}$ particle in Particle Swarm Optimization Algorithm 


\section{References}

Abido, M. A. (2002). Optimal design of power-system stabilizers using particle swarm optimization. IEEE transactions on energy conversion, 17(3), 406-413.

Bansal, J. C., Singh, P. K., Saraswat, M., Verma, A., Jadon, S. S., \& Abraham, A. (2011, October). Inertia weight strategies in particle swarm optimization. In Nature and Biologically Inspired Computing (NaBIC), 2011 Third World Congress on (pp. 633-640). IEEE.

Clerc, M. (2010). Particle swarm optimization (Vol. 93). John Wiley \& Sons.

Clerc, M., \& Kennedy, J. (2002). The particle swarm-explosion, stability, and convergence in a multidimensional complex space. IEEE transactions on Evolutionary Computation, 6(1), 58-73.

Chan, F. T. S., \& Tiwari, M. K (2007). Swarm Intelligence: focus on ant and particle swarm optimization. I-Tech Education and Publishing. Cited on, 146.

Del Valle, Y., Venayagamoorthy, G. K., Mohagheghi, S., Hernandez, J. C., \& Harley, R. G. (2008). Particle swarm optimization: basic concepts, variants and applications in power systems. IEEE Transactions on evolutionary computation, 12(2), 171-195.

Di Cesare, N., Chamoret, D., \& Domaszewski, M. (2015). A new hybrid PSO algorithm based on a stochastic Markov chain model. Advances in Engineering Software, 90, 127-137.

Eberhart, R., \& Kennedy, J. (1995). A new optimizer using particle swarm theory. In Micro Machine and Human Science, 1995. MHS'95., Proceedings of the Sixth International Symposium on (pp. 39-43). IEEE.

Eberhart, R. C., Shi, Y., \& Kennedy, J. (2001). Swarm Intelligence (The Morgan Kaufmann Series in Evolutionary Computation).

Kacprzyk, J. (2009). Studies in Computational Intelligence, Volume 198.

Lee, K. Y., \& Park, J. B. (2006, October). Application of particle swarm optimization to economic dispatch problem: advantages and disadvantages. In Power Systems Conference and Exposition, 2006. PSCE'06. 2006 IEEE PES (pp. 188-192). IEEE. 
Mavrovouniotis, M., Li, C., \& Yang, S. (2017). A survey of swarm intelligence for dynamic optimization: algorithms and applications. Swarm and Evolutionary Computation, 33, 1-17.

Poli, R., Kennedy, J., \& Blackwell, T. (2007). Particle swarm optimization. Swarm intelligence, 1(1), 33-57.

Shi, Y., \& Eberhart, R. (1998, May). A modified particle swarm optimizer. In Evolutionary Computation Proceedings, 1998. IEEE World Congress on Computational Intelligence., The 1998 IEEE International Conference on (pp. 69-73). IEEE.

Ting, T. O., Shi, Y., Cheng, S., \& Lee, S. (2012, June). Exponential inertia weight for particle swarm optimization. In International Conference in Swarm Intelligence (pp. 83-90). Springer, Berlin, Heidelberg. 


\section{Figures}

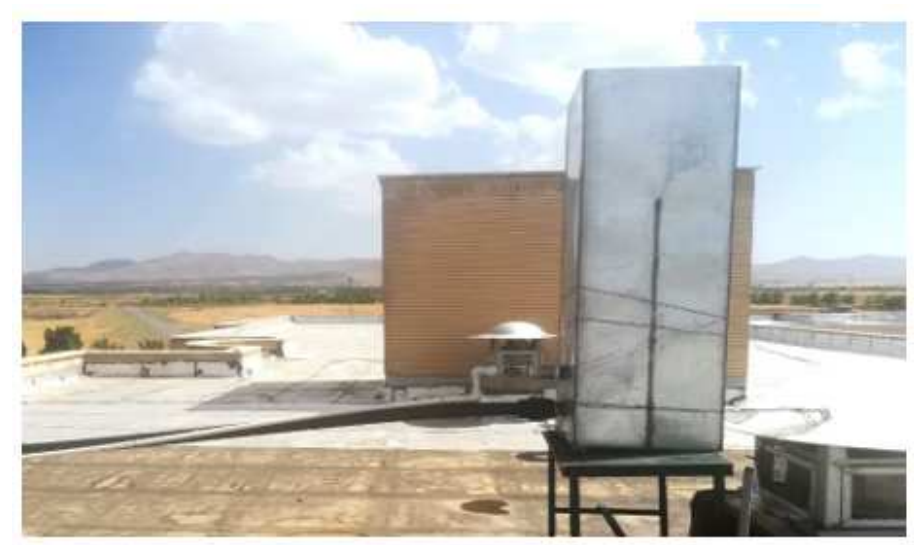

a

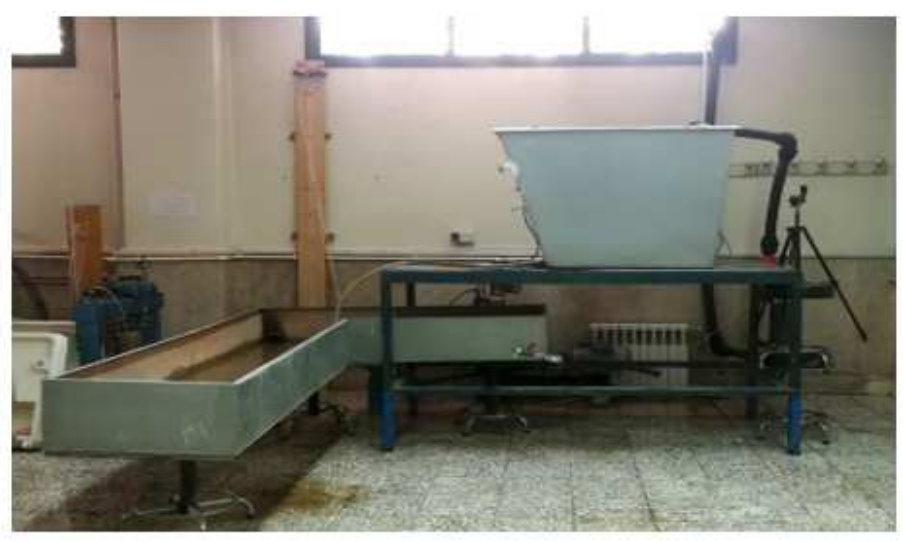

b

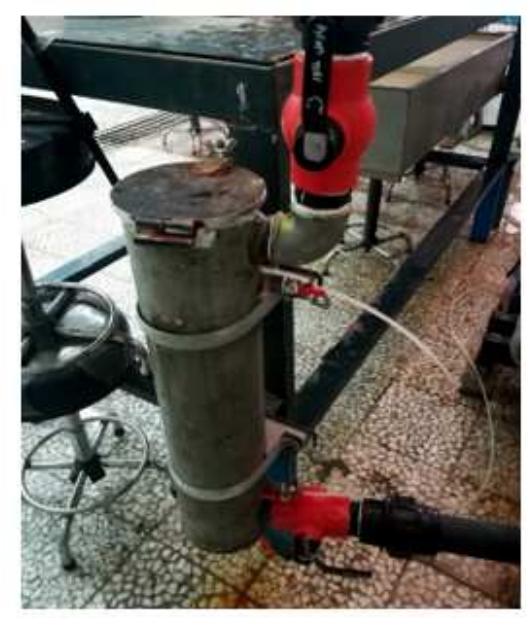

c

\section{Figure 1}

Schematic view of the experimental setup, a) the installed tank on the roof, b) the tank and cylinder installed in the laboratory, and c) steel cylinder 


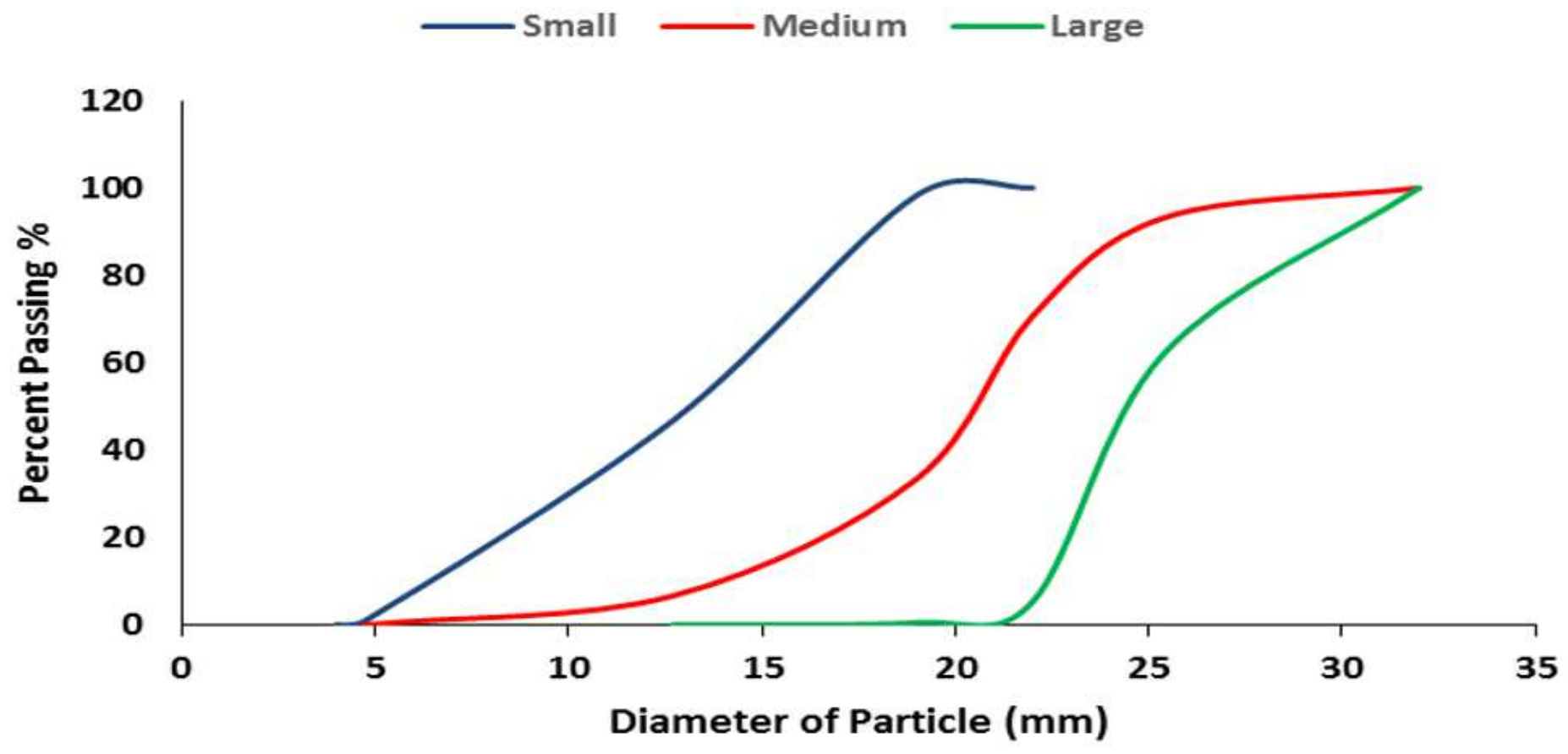

Figure 2

Gradation curve of different materials 


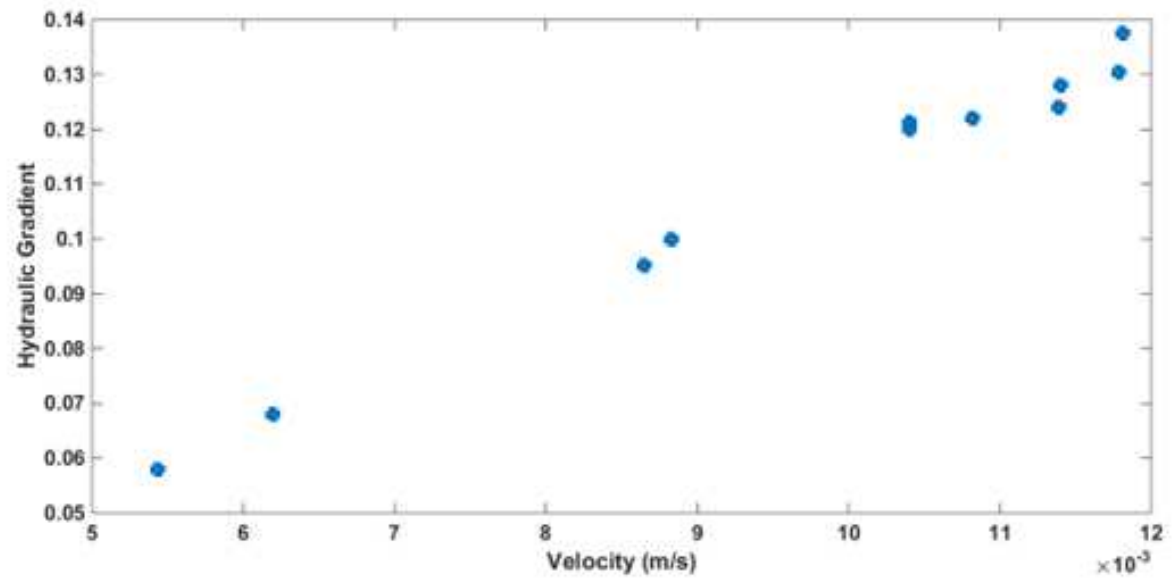

a. Small
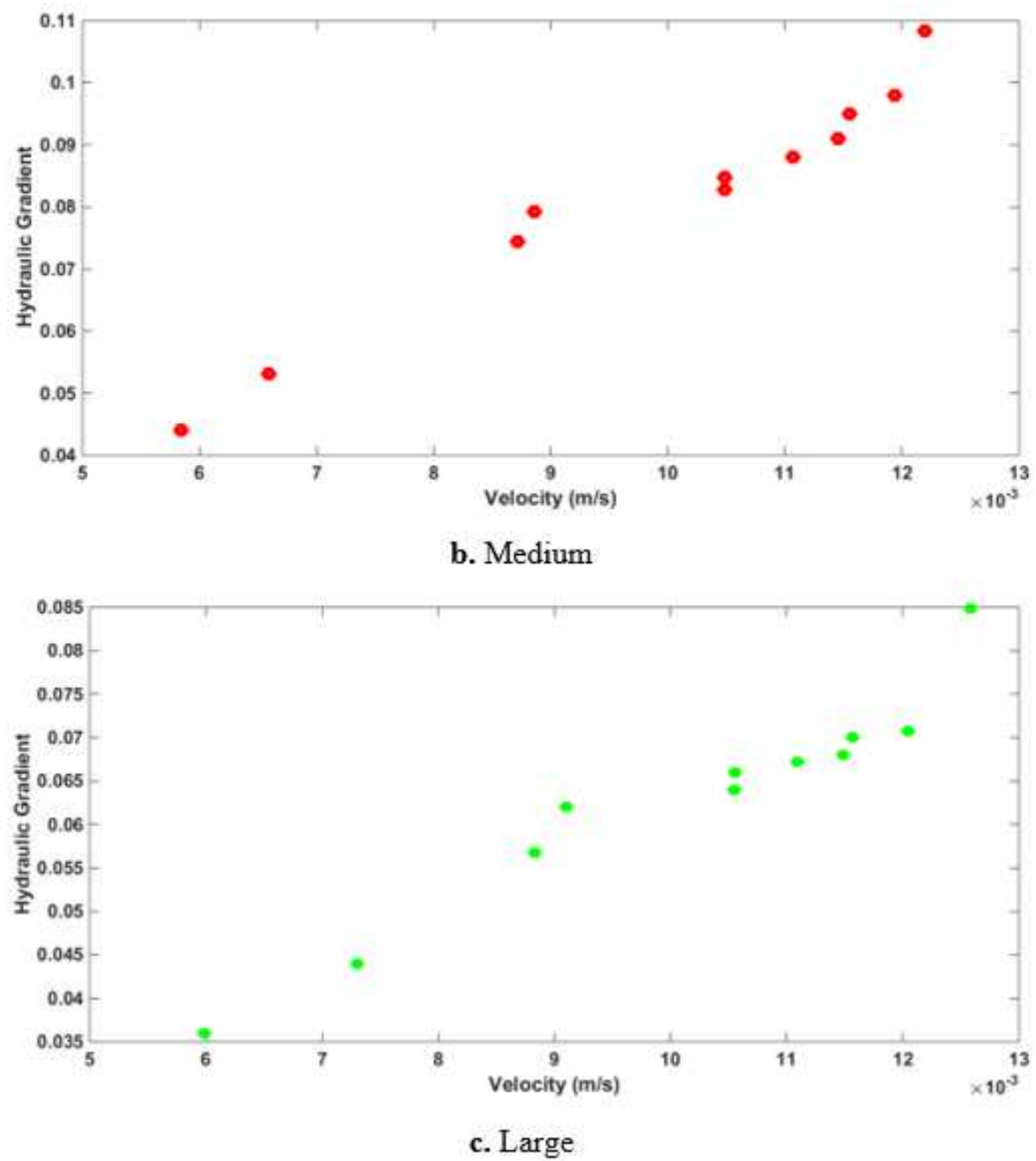

Figure 3

Changes in hydraulic gradient versus steady flow velocity recorded in the laboratory 


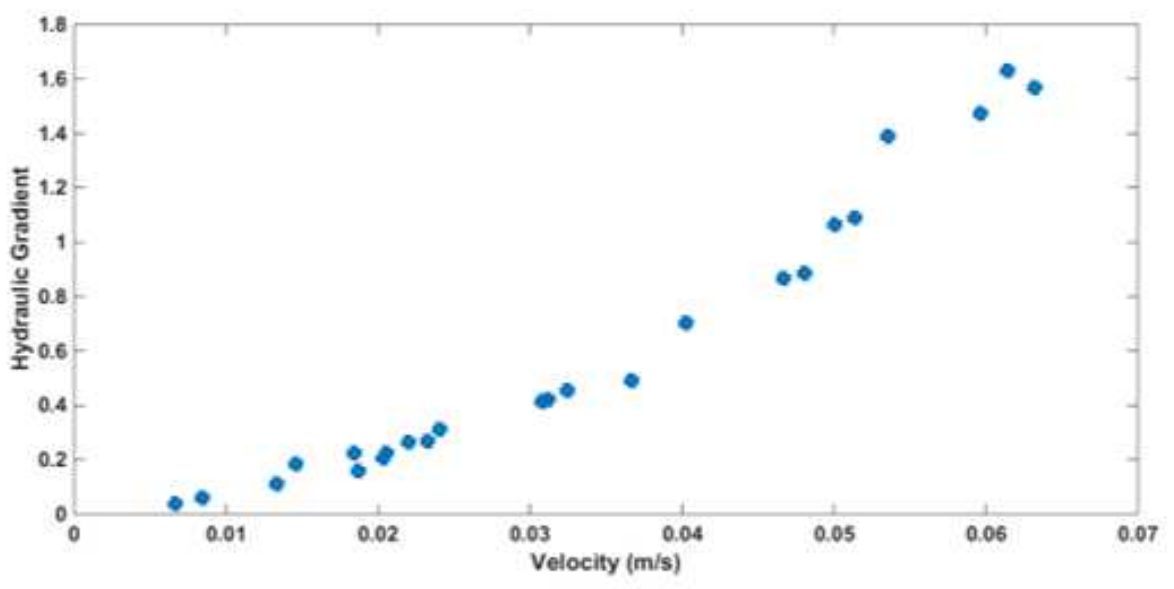

a. Small

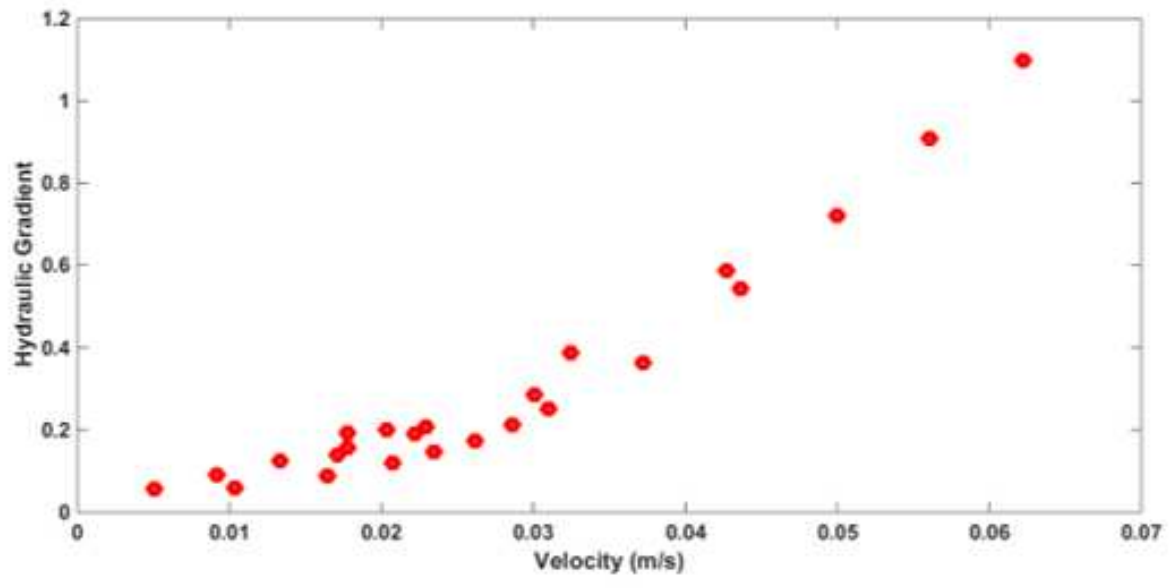

b. Medium

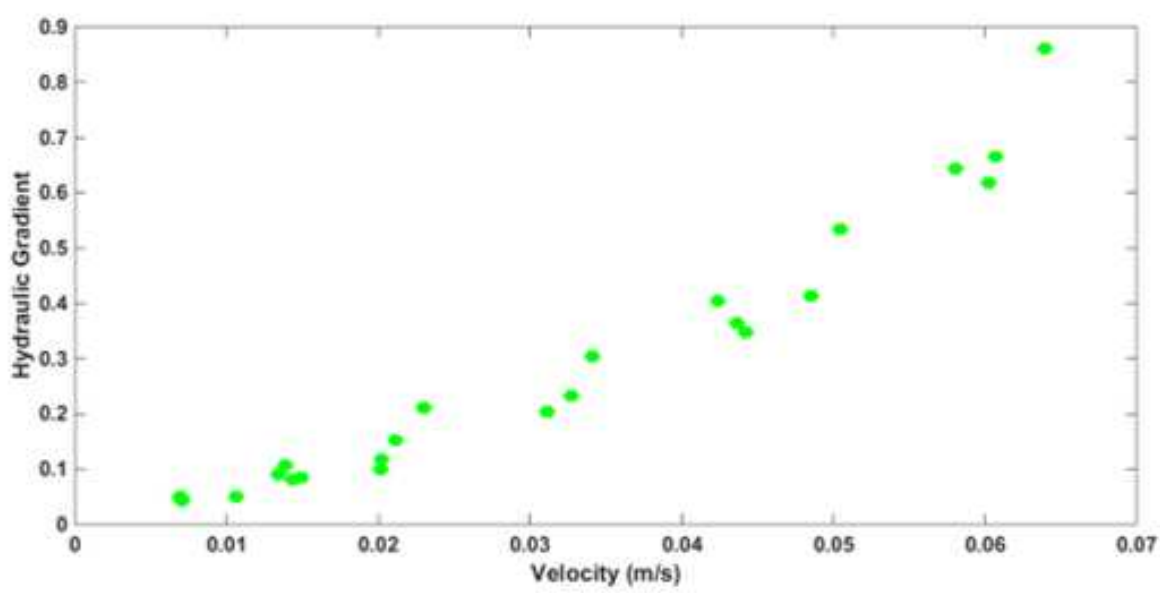

c. Large

Figure 4

Changes in hydraulic gradient versus unsteady flow velocity recorded in the laboratory 


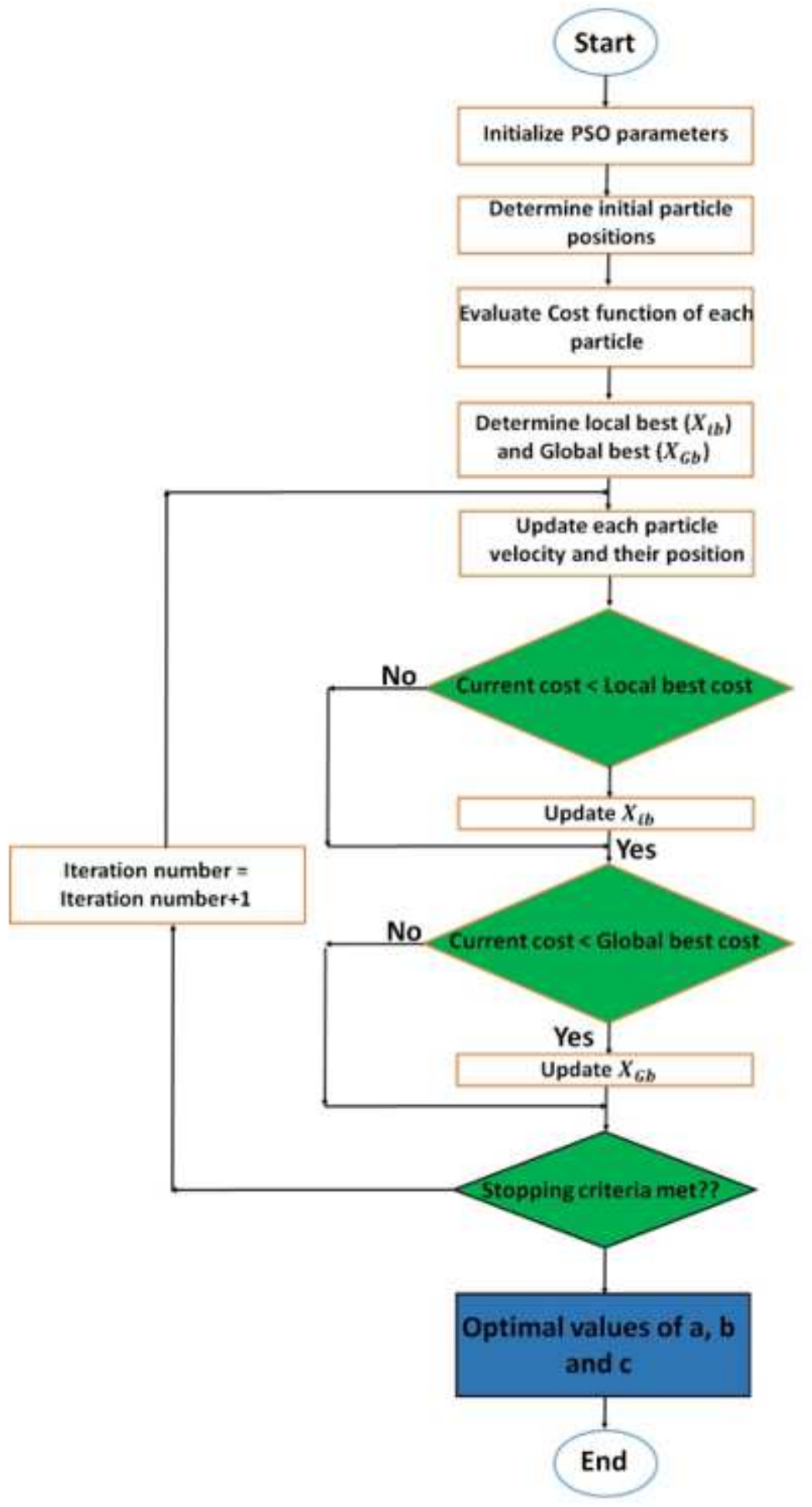

Figure 5

Particle Swarm Optimization (PSO) algorithm 


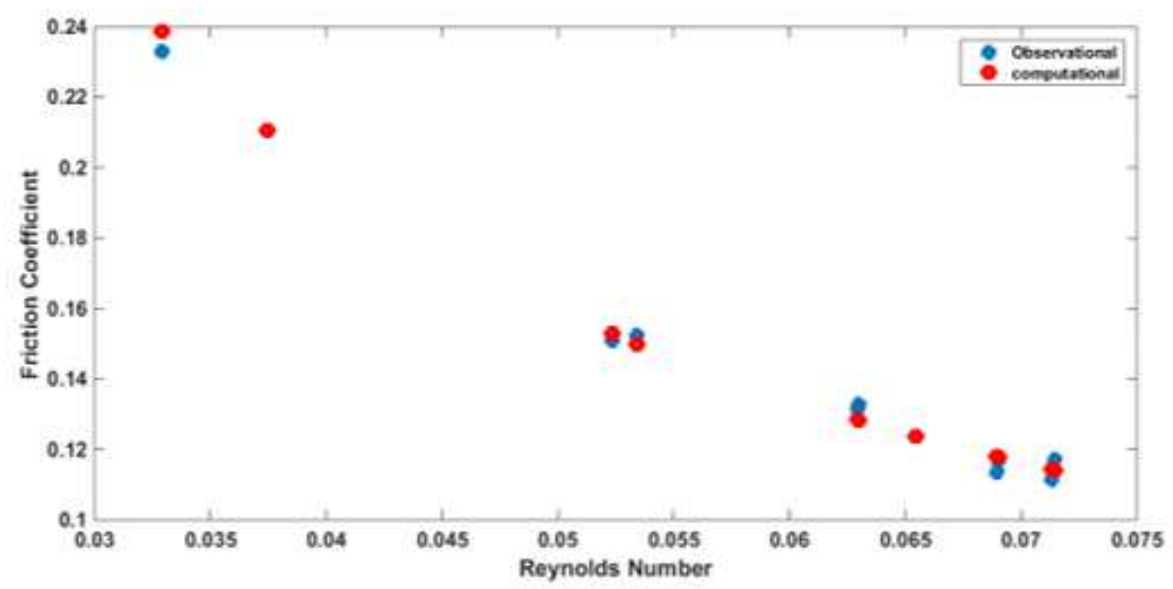

a. Small

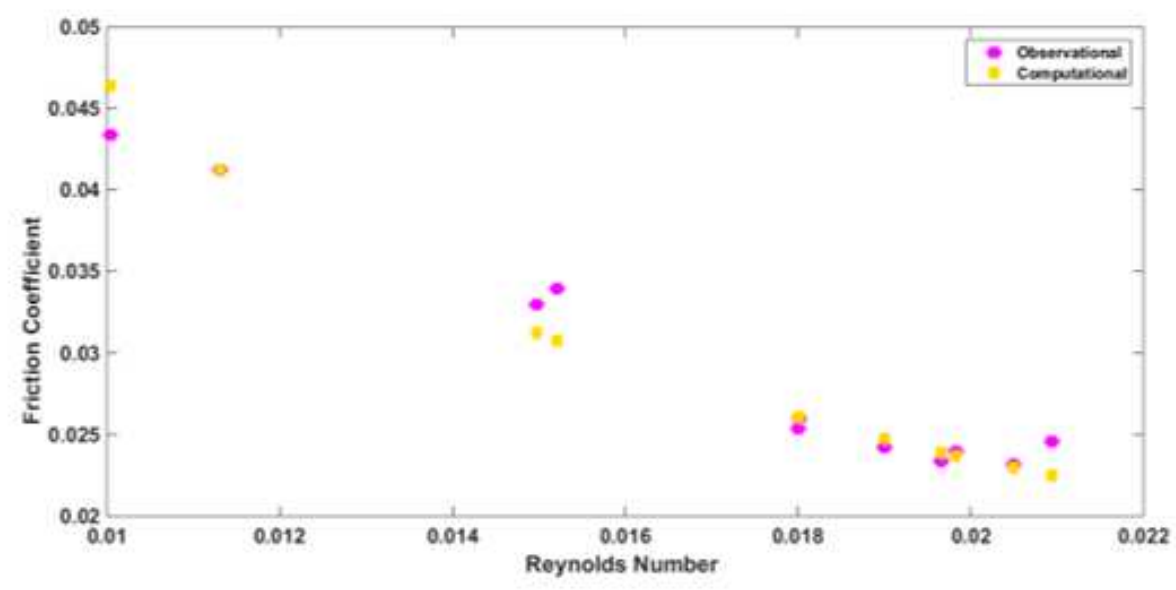

b. Medium

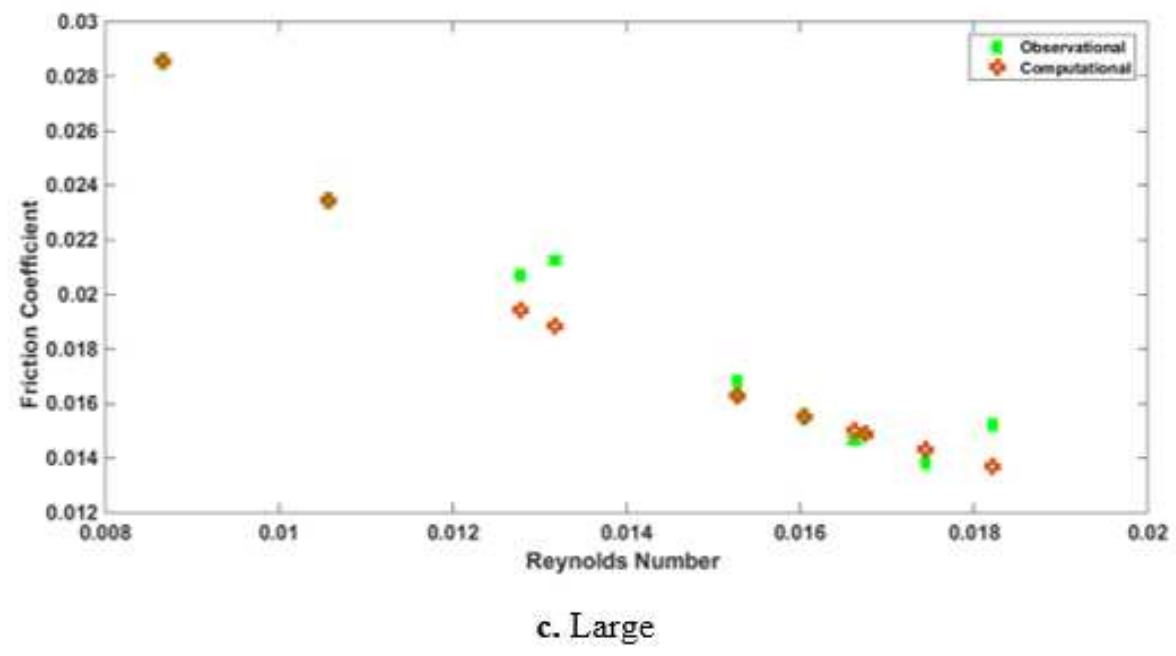

Figure 6

Observational and computational friction coefficient versus Reynolds number in steady flow condition 


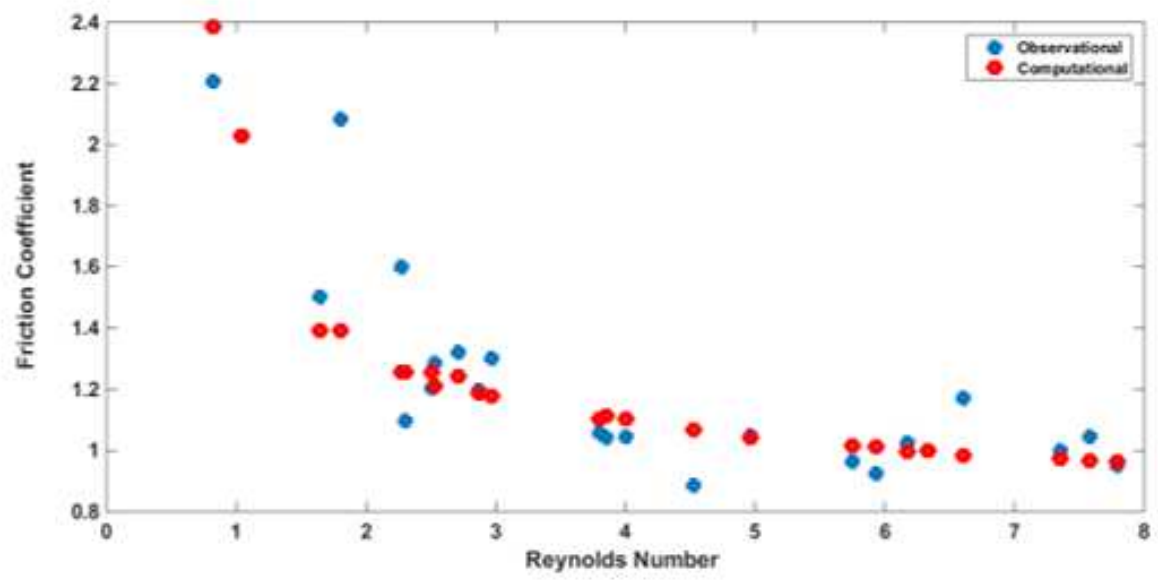

a. Small

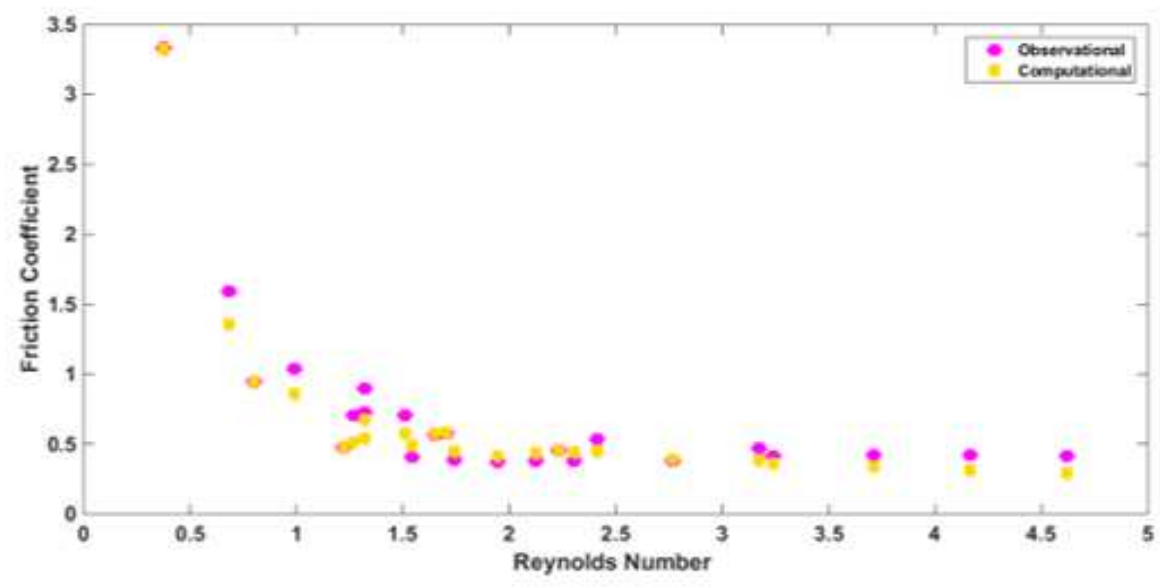

b. Medium

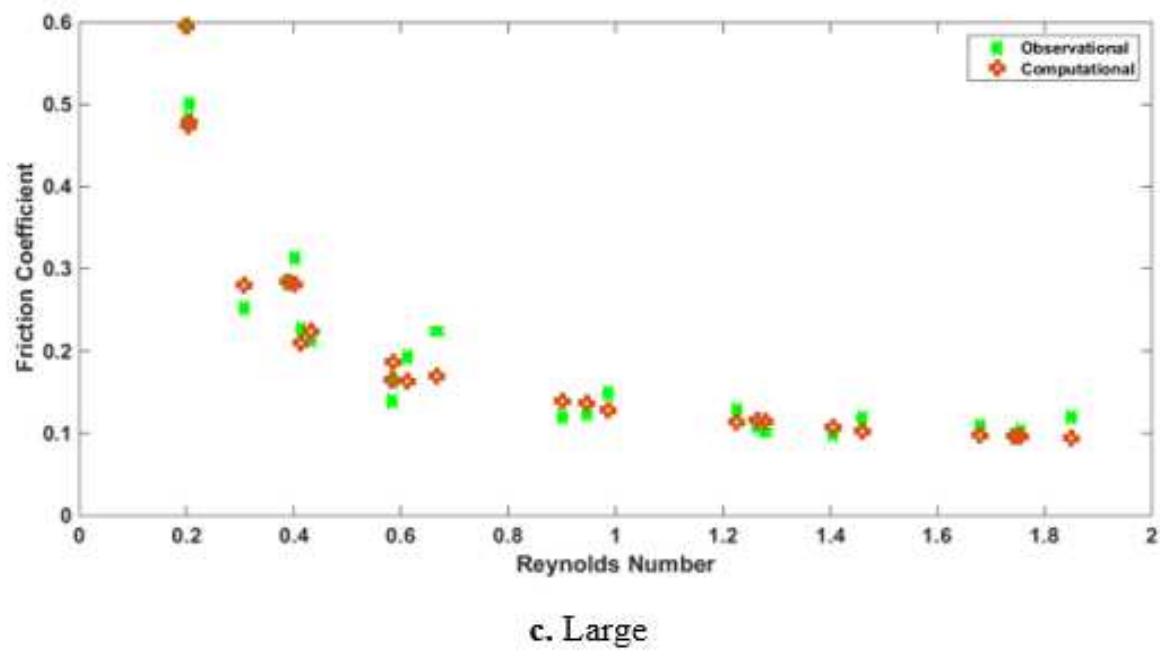

Figure 7

Changes in observational and computational friction coefficients in terms of Reynolds number in unsteady flow condition 